ARTICLE

https://doi.org/10.1038/s41467-019-12726-0

\title{
Functional profiling of single CRISPR/Cas9-edited human long-term hematopoietic stem cells
}

Elvin Wagenblast (10 ${ }^{1}$, Maria Azkanaz ${ }^{1}$, Sabrina A. Smith ${ }^{1}$, Lorien Shakib ${ }^{1}$, Jessica L. McLeod', Gabriela Krivdova ${ }^{1}$ Joana Araújo1, Leonard D. Shultz² ${ }^{2}$ Olga I. Gan¹, John E. Dick (iD ${ }^{1,3 \star}$ \& Eric R. Lechman ${ }^{1 \star}$

In the human hematopoietic system, rare self-renewing multipotent long-term hematopoietic stem cells (LT-HSCs) are responsible for the lifelong production of mature blood cells and are the rational target for clinical regenerative therapies. However, the heterogeneity in the hematopoietic stem cell compartment and variable outcomes of CRISPR/Cas9 editing make functional interrogation of rare LT-HSCs challenging. Here, we report high efficiency LT-HSC editing at single-cell resolution using electroporation of modified synthetic gRNAs and Cas9 protein. Targeted short isoform expression of the GATA1 transcription factor elicit distinct differentiation and proliferation effects in single highly purified LT-HSC when analyzed with functional in vitro differentiation and long-term repopulation xenotransplantation assays. Our method represents a blueprint for systematic genetic analysis of complex tissue hierarchies at single-cell resolution.

\footnotetext{
${ }^{1}$ Princess Margaret Cancer Centre, University Health Network, Toronto, ON M5G 1L7, Canada. ${ }^{2}$ The Jackson Laboratory, Bar Harbor, ME 04609 , USA. ${ }^{3}$ Department of Molecular Genetics, University of Toronto, Toronto, ON M5S 1A8, Canada. These authors contributed equally: John E. Dick, Eric R. Lechman. *email: john.dick@uhnresearch.ca; elechman@uhnresearch.ca
} 
T he hematopoietic stem and progenitor cell (HSPC) compartment is a functional continuum comprised of multiple stem and progenitor cell populations including abundant committed progenitors such as common myeloid progenitors and myelo-erythroid progenitors (MEPs), as well as rarer multipotent stem cells including short-term hematopoietic stem cells (STHSCs) and long-term hematopoietic stem cells (LT-HSCs) ${ }^{1,2}$ (Supplementary Fig. 1). LT-HSCs are the only population that have the ability to permanently repopulate the entire hematopoietic system following transplantation ${ }^{2}$; they represent the key target for blood-based regenerative therapies. Thus, LT-HSCs are essential for therapeutic genome editing to correct acquired and genetic hematopoietic disorders ${ }^{3,4}$. Furthermore, the pathogenesis of hematological malignancies like acute myeloid leukemia (AML) is associated with the presence of initiating mutations acquired in LT-HSCs, which lead to their competitive expansion $^{5,6}$. Pre-leukaemic LT-HSCs are a source of clonal evolution within blood malignancies and can act as a reservoir of relapse after chemotherapy treatment ${ }^{7}$. Previous studies have shown the feasibility of using genome editing techniques in human CD34 ${ }^{+}$ cells to model hematological diseases, such as using CRISPR/Cas9 to induce myeloid neoplasia ${ }^{8}$ or using transcription activator-like effector nucleases to induce chromosomal translocations to model MLL-rearranged leukemia ${ }^{9}$. Thus, modeling and understanding the genetic complexity and cellular heterogeneity seen in human hematological malignancies using novel methodologies that allow genome editing in highly purified single LT-HSCs and their functional read-out are of great need ${ }^{3,4}$.

Recently several studies have demonstrated efficient gene editing of bulk $\mathrm{CD} 34^{+}$populations that are enriched for human HSPCs $8,10-18$. Highly efficient non-homologous end joining (NHEJ)-mediated gene disruption of up to $80-90 \%$ efficiency has been reported in CD34 ${ }^{+} \mathrm{HSPCs}^{12,13,15,17}$. In addition, homologydirected repair (HDR)-mediated knock-ins, with or without selectable fluorescent reporter genes, have been established with an efficiency of up to $20 \%$ in CD34 $4^{+}$HSPCs $^{10,11,13,14,16,18}$. Stable integration of a fluorescent reporter using rAAV6 combined with flow cytometry-based sorting enabled enrichment of CRISPR/ Cas9-edited HSPCs ${ }^{10,11,17}$. Because LT-HSCs represent only $0.1-1 \%$ of $\mathrm{CD} 34^{+}$populations, these studies did not address LTHSC targeting in the most direct manner. Previous studies have reported long-term engraftment of up to 16 weeks following xenotransplantation of CRISPR/Cas9 edited human $\mathrm{CD} 34^{+}$ HSPCs $^{10,16,18}$, suggesting that rare LT-HSCs within the CD $34^{+}$ population can be gene edited. However, these studies utilized considerable numbers of $\mathrm{CD} 34^{+}$HSPCs and lacked the resolution to functionally interrogate the differentiation and proliferation properties of individual LT-HSCs. In order to simplify LTHSC targeting within heterogeneous CD34 ${ }^{+}$HSPCs, we explored the possibility of CRISPR/Cas9 editing in highly purified LTHSCs. This approach would enable direct functional characterization of LT-HSCs, rather than bulk populations. Here, we show successful editing of highly purified LT-HSCs via CRISPR/Cas9mediated NHEJ or HDR and their subsequent functional investigation using single cell in vitro differentiation and near-clonal xenotransplantation assays.

\section{Results}

CRISPR/Cas9-mediated GATA1 isoform expression in LTHSCs. As a proof of principle, we modeled GATA1 isoform expression in LT-HSCs, ST-HSCs, and MEPs from neonatal cord blood using CRISPR/Cas9. GATA1 encodes a DNA binding protein with two zinc fingers and a transactivation domain that is required for erythroid, megakaryocyte, mast cell, eosinophil, and basophil differentiation ${ }^{19-21}$. Acquired and inherited GATA1 mutations contribute to hematological disorders such as Down syndrome acute megakaryoblastic leukemia (AMKL), DiamondBlackfan anemia, transient myeloproliferative disorder and congenital dyserythropoietic anemias with thrombocytopenia ${ }^{22-27}$. The GATA1 gene normally produces two protein isoforms as a result of alternative mRNA splicing-the GATA1 full length (GATA1-Long) and a truncated form (GATA1-Short). GATA1 isoform biology is particularly important for children with Down syndrome and a subset of children born with Diamond-Blackfan anemia. Children with Down syndrome have a 150 -fold higher risk of developing AMKL, which is characterized by an abnormal proliferation of immature megakaryocytes ${ }^{28-30}$. Mutations in exon 2, which lead to the exclusive expression of GATA1-Short, are thought to be an essential driver of this disease. Previous work has shown effects of GATA1-Short on megakaryocytic proliferation, but these changes were only seen in fetal HSPCs and not in neonatal or adult HSPCs, implying a developmental stagespecific effect ${ }^{31,32}$. To verify this hypothesis, we decided to test GATA1-Long versus GATA1-Short isoform expression in purified LT-HSCs, ST-HSCs, and MEPs from neonatal cord blood.

Overall, our experimental scheme employed flow cytometric isolation of cord blood LT-HSCs for xenotransplantation and isolation of LT-HSCs, ST-HSCs, and MEPs for single cell in vitro differentiation assays (Supplementary Fig. 2). Sorted cells were cultured for $48 \mathrm{~h}$ and electroporated with modified synthetic gRNAs and Cas9 protein (Fig. 1a). During brief in vitro culturing, LT-HSCs durably retain their immuno-phenotype with some variability seen in ST-HSCs and MEP subsets. (Supplementary Fig. 3). Because of the transient delivery of the ribonucleoprotein complex and lack of a selectable marker, all CRISPR/Cas9mediated edits were subsequently genetically verified. To express the GATA1-Short isoform, we used two gRNAs targeting the 5' and $3^{\prime}$ flanking regions of exon 2, resulting in the NHEJ-mediated dropout of the exon (Fig. 1b). By contrast, mutation of the GATA1-Short alternative start site on exon 3 from ATG to CTC via CRISPR/Cas9-mediated HDR led to the exclusive expression of the GATA1-Long isoform (Fig. 1c). Importantly, expression of both the GATA1-Short and -Long isoforms remained under the regulatory control of the endogenous GATA1 promoter. Because GATA1 is X-linked, all our studies utilized male cord blood samples. As a control, we used two gRNAs targeting exon 1 of the olfactory receptor OR2W5 that were designed with the CRoatan algorithm ${ }^{33}$, resulting in a $150 \mathrm{bp}$ dropout of the exon. After electroporation, individual LT-HSCs, ST-HSCs, and MEPs were deposited into single cell in vitro assays under erythro-myeloid differentiation conditions[34] (Fig. 1a). After 16-17 days, each single cell-derived colony was assessed by flow cytometry for lineage output and the genotype of GATA1-Short or control edited cells was determined by polymerase chain reaction (PCR), whereas the genotype of GATA1-Long edited cells was assessed by Sanger sequencing (Supplementary Fig. 4a-c). Moreover, LTHSCs that were CRISPR/Cas9 edited with GATA1-Short or control gRNAs were transplanted into mice at a near-clonal level in order to detect lineage and proliferation biases (Fig. 1a).

Single-cell differentiation of CRISPR/Cas9-edited LT-HSCs. CRISPR/Cas9 editing efficiency in LT-HSCs, ST-HSCs, and MEPs was high; the percentage of single cell-derived colonies with homozygous deletion of OR2W5, GATA1-Short, and GATA1Long was $50-60 \%, 40 \%$, and $20 \%$, respectively (Fig. $2 \mathrm{a}$ and Supplementary Fig 5a). Any control or GATA1-Short edited single cell colony with only one gRNA cut and no exon dropout was disregarded in the initial analysis. Although statistically insignificant, CRISPR/Cas9-mediated HDR efficiencies were lower in LT-HSCs than in ST-HSCs and MEPs (Fig. 2a). While 
a

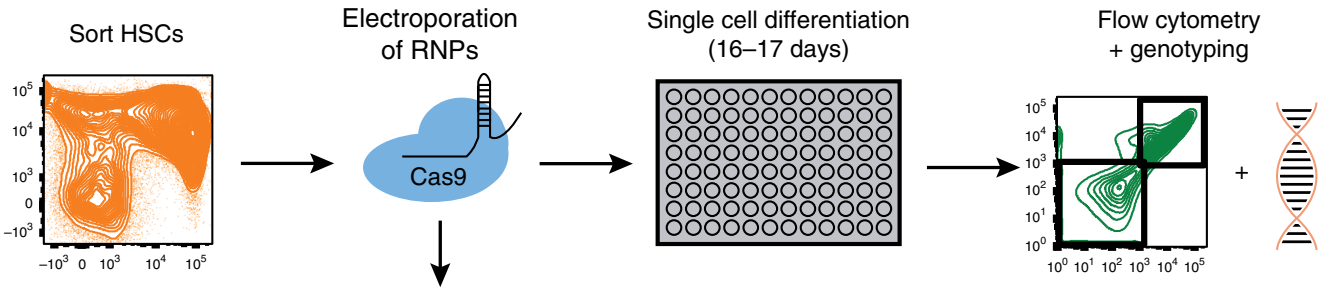

Near-clonal xenotransplantation (12/24 weeks)
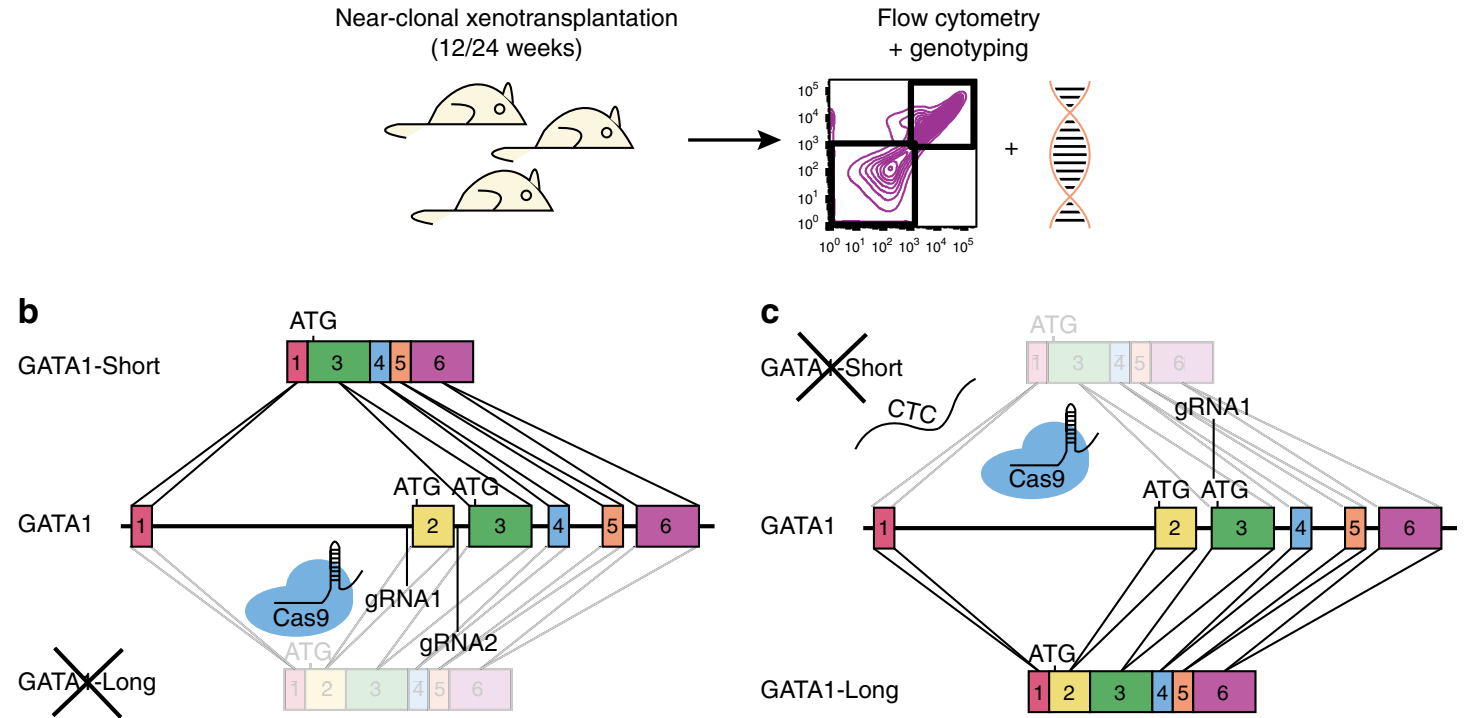

Fig. 1 CRISPR/Cas9-mediated isoform expression of GATA1 at single cell level. a Experimental workflow for single cell in vitro differentiation assay and near-clonal xenotransplantation into mice. b Two gRNAs targeting the $5^{\prime}$ and $3^{\prime}$ end of exon 2 of GATA1 led to the NHEJ-mediated dropout of this exon, resulting in the exclusive expression of GATA1-Short. c HDR-mediated mutation of the alternative start site from ATG to CTC using a single gRNA and a single-strand DNA template, resulting in the exclusive expression of GATA1-Long

electroporation by itself did not drastically affect the efficiency of single cells to form colonies, LT-HSCs had slightly lower single cell colony formation efficiencies compared to ST-HSCs and MEPs (Fig. 2b). No off-target cleavage was detected at loci that were similar in sequence to the gRNA target sequence by amplicon Sanger sequencing (Supplementary Fig. 5b). Although no whole genome sequencing was performed, the likelihood that these results are due to off-target cleavage is extremely low. In addition, karyotyping analysis revealed no structural abnormalities after CRISPR/Cas9 editing in any of the conditions (Supplementary Fig. 5c). Western assay of bulk CRISPR/Cas9-edited MEPs cultured under erythro-myeloid conditions showed enrichment of either the GATA1-Long or GATA1-Short isoform (Fig. 2c, Supplementary Fig. 6a).

Culture of single LT-HSC, ST-HSC, and MEP under erythromyeloid differentiation conditions revealed a drastic shift towards megakaryocytic lineage output upon exclusive expression of GATA1-Short, with a twofold and fourfold increase in $\mathrm{CD} 41^{+}$ megakaryocytic colonies compared to LT-HSCs expressing control and GATA1-Long, respectively (Fig. 2d, e). Interestingly, only GATA1-Short edited LT-HSCs produced bi-potent myelomegakaryocytic colonies. ST-HSCs showed even higher fold increases toward megakaryocytic lineage output compared to LTHSCs. Whereas CRISPR/Cas9 control edited MEPs did not possess any megakaryocytic differentiation capacity, GATA1Short edited MEPs were able to produce $\mathrm{CD} 41^{+}$megakaryocytes, albeit with lower efficiency compared to LT-HSCs and ST-HSCs. In order to mimic precise mutations at the $5^{\prime}$ splice junction of exon 2 of patients with Down Syndrome associated leukemia ${ }^{26}$, single-cell derived colonies were analyzed where only 1 gRNA was utilized in order to target the $5^{\prime}$ splice site of exon 2 (Supplementary Fig. 6b-d). Similarly, there is an increase in the number of megakaryocytic lineage positive colonies in GATA1 splice junction edited LT-HSCs (M, Meg) and STHSCs and MEPs (M, E, Meg) compared to control-edited colonies. In addition to the engineering of CRISPR/Cas9mediated isoform re-arrangements, single gRNA mediated knock-outs can also be utilized in our method; for example, they can be used against STAG2 with single cell CRISPR/Cas9 efficiencies as high as $80-90 \%$ in LT-HSCs (Supplementary Fig. 6e, f). In summary, purified LT-HSCs and more committed stem and progenitor cells can be edited with high efficiency by CRISPR/Cas9-mediated NHEJ and HDR, and the effects of gene editing on differentiation can be read out reliably using single cell in vitro assays.

Long-term xenotransplantation of CRISPR/Cas9-edited LTHSCs. Human LT-HSC can only be evaluated functionally using the gold standard xenograft assay ${ }^{35}$. To investigate the functional consequences of exclusive GATA1-Short expression in LT-HSCs in vivo, we performed near-clonal xenotransplantation assays in NSG mice. Limiting dilution analysis ${ }^{36}$ of CRISPR/Cas9 controledited LT-HSCs injected into NSG mice for 24 weeks revealed a repopulating stem cell frequency of $\sim 1 / 100$ edited cells (Supplementary Fig. 7a). To achieve near-clonal xenotransplantation, we transplanted control- or GATA1-Short-edited LT-HSCs into NSG mice at an equivalent dose of 100-150 cells/mouse and after 24 weeks analyzed bone marrow (BM) cells harvested from the injected right femur (RF) and the left femur plus both tibias (BM). Only mice with human $\mathrm{CD}^{2} 5^{+}$engraftment levels of $>5 \%$ in the RF and $>90 \%$ CRISPR/Cas9 editing efficiency as determined by PCR and Sanger sequencing were included in our analysis: $40 \%$ of control mice and $35 \%$ of mice transplanted with 
a

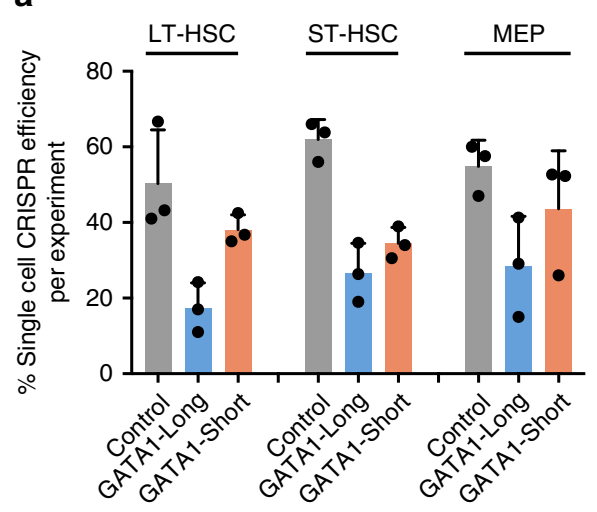

d b

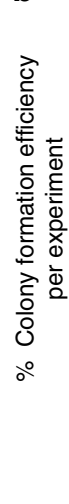

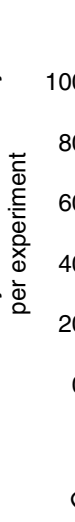

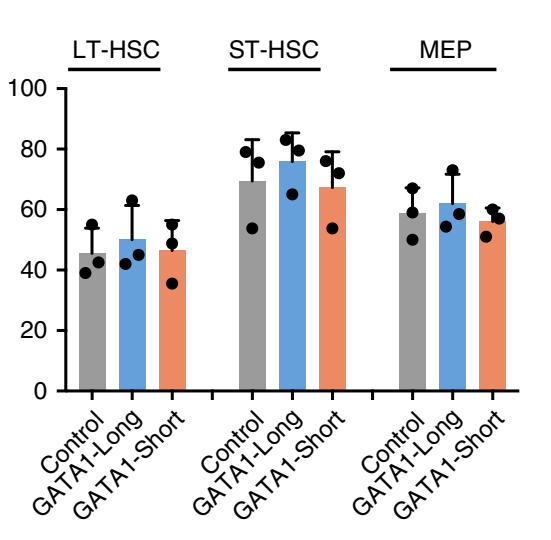

e
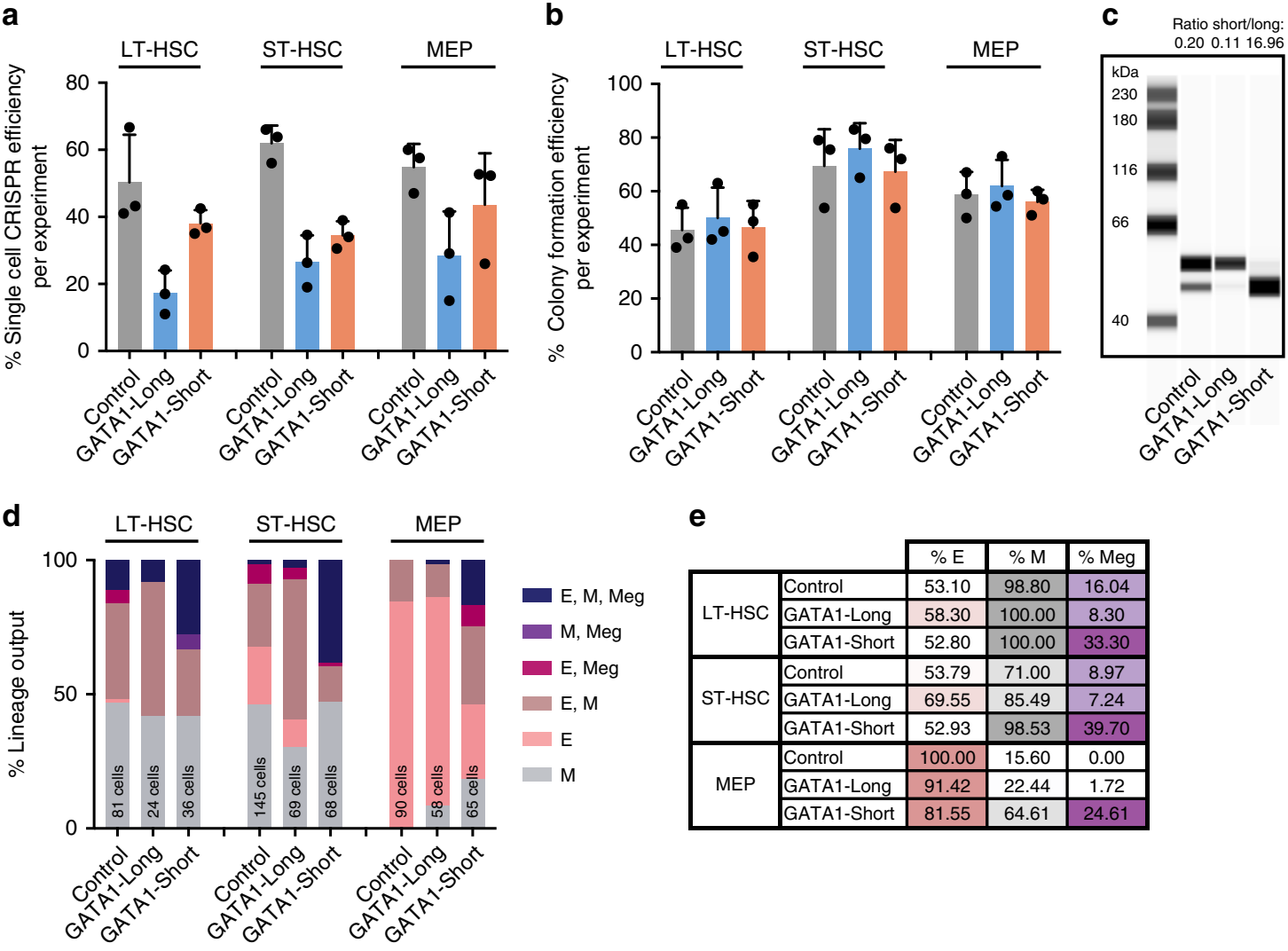

\begin{tabular}{|c|l|c|c|c|}
\cline { 2 - 5 } \multicolumn{2}{c|}{} & $\% \mathrm{E}$ & $\% \mathrm{M}$ & $\% \mathrm{Meg}$ \\
\hline \multirow{3}{*}{ LT-HSC } & Control & 53.10 & 98.80 & 16.04 \\
\cline { 2 - 5 } & GATA1-Long & 58.30 & 100.00 & 8.30 \\
\cline { 2 - 5 } & GATA1-Short & 52.80 & 100.00 & 33.30 \\
\hline \multirow{3}{*}{ ST-HSC } & Control & 53.79 & 71.00 & 8.97 \\
\cline { 2 - 5 } & GATA1-Long & 69.55 & 85.49 & 7.24 \\
\cline { 2 - 5 } & GATA1-Short & 52.93 & 98.53 & 39.70 \\
\hline \multirow{3}{*}{ MEP } & Gontrol & 100.00 & 15.60 & 0.00 \\
\cline { 2 - 5 } & GATA1-Long & 91.42 & 22.44 & 1.72 \\
\cline { 2 - 5 } & GATA1-Short & 81.55 & 64.61 & 24.61 \\
\hline
\end{tabular}

Fig. 2 Functional interrogation of single CRISPR/Cas9-edited hematopoietic stem cells in vitro. a Percentage of CRISPR/Cas9 efficiency as determined by single cell-derived colonies that were positive for homozygous deletion of exon 1 in control OR2W5, positive for exclusive assignment to the GATA1-Long isoform through HDR-mediated mutation of the alternative start site or positive for exclusive assignment to the GATA1-Short isoform through deletion of exon 2 ( $n=3$ experiments with independent cord blood pools). b Percentage of single cells that grew into a colony in the single cell in vitro differentiation assays ( $n=3$ experiments with independent cord blood pools, unpaired $t$ test $P<0.05$ for \% colony efficiency in LT-HSCs GATA1-Long versus ST-HSCs GATA1-Long). c Capillary-based western assay of GATA1 in bulk MEPs that were CRISPR/Cas9 edited with control, GATA1-Long and GATA1-Short gRNAs and cultured under erythro-myeloid conditions for three days ( $n=2$ experiments, second western assay is shown in Supplementary Fig. 6a). d Lineage output from the in vitro single cell differentiation assay of individual CRISPR/Cas9-edited LT-HSCs, ST-HSCs and MEPs with control, GATA1-Long and GATA1-Short gRNAs. Numbers of single cell colonies with positive genotype are indicated at each condition $(E=$ erythroid, $M=$ myeloid, Meg $=$ megakaryocytic, $n=3$ experiments with independent cord blood pools, number of single cells assessed in each condition is indicated in each bar graph, unpaired $t$ test $P<0.01$ for $E, M$, Meg in GATA1-Short versus control and E, M, Meg in GATA1-Short versus GATA1-Long among all three cell types). e Overall percentage of erythroid, myeloid and megakaryocyte containing colonies from single cell in vitro differentiation assays ( $E=$ erythroid, $M=$ myeloid, Meg = megakaryocytic, $n=3$ experiments with independent cord blood pools, unpaired $t$ test $P<0.005$ for $\%$ Meg in GATA1-Short versus Control or \% Meg in GATA1-Short versus GATA1-Long among all three cell types). Error bars represent standard deviations

GATA1-Short edited LT-HSCs fulfilled these criteria (Fig. 3a, Supplementary Fig. $7 \mathrm{~b}-\mathrm{f}$ ). To precisely determine the genotype of the clonal progeny of injected LT-HSCs, secondary methylcellulose colony formation assays from cells of the RF were carried out. Analysis of CRISPR/Cas9 edits in individual colonies by Sanger sequencing revealed clonal engraftment in 3 out of 5 GATA1-Short edited LT-HSCs injected mice, highlighting that the xenotransplantations were indeed performed at near clonal levels (Supplementary Fig. 7g). On average, human $\mathrm{CD}_{4}{ }^{+}$ engraftment in the RF was $40 \%$, both in control and GATA1Short edited LT-HSCs injected mice (Fig. 3b). Interestingly, GATA1-Short edited LT-HSCs generated grafts with twofold higher percentage of human $\mathrm{CD} 41^{+} \mathrm{CD} 45^{-}$megakaryocytic lineage derived cells in the RF compared to controls (Fig. 3c). In addition, we observed an increase in the percentage of human CD $19^{+}$CD $45^{+}$B-lymphoid cells in the RF (Fig. 3d, Supplementary Fig. 8a-d). GATA1-Short edited LT-HSCs generated grafts with higher absolute cell numbers, mainly due to increased numbers of B-lymphoid lineage cells (Fig. 3e, Supplementary Fig. 8e-i). The observed increase in megakaryocytic lineage output in mice transplanted with GATA1-Short edited LT-HSCs (Fig. 3f) confirms our single cell in vitro findings, and demonstrates the feasibility of conducting near-clonal xenotransplantation assays using purified CRISPR/Cas9-edited LT-HSCs.

Normal human HSCs show a predominant B-lymphoid bias upon long-term engraftment in NSG mice ${ }^{37}$. We therefore repeated our xenotransplantation assays using c-kit-deficient NSGW41 recipients, which support enhanced erythropoietic and megakaryocytic lineage output ${ }^{38}$. Limiting dilution analysis of CRISPR/Cas9-edited LT-HSCs in NSGW41 mice for 12 weeks revealed a repopulating cell frequency of $\sim 1 / 175$ (Supplementary Fig. 9a). Consequently, an equivalent dose of 200-250 control- or GATA1-Short-edited LT-HSCs were transplanted into NSGW41 mice and human engraftment in the RF and BM was analyzed after 12 weeks. Totally, $35 \%$ of mice transplanted with control edited LT-HSCs and $30 \%$ of mice transplanted with GATA1Short edited LT-HSCs showed robust engraftment and successful CRISPR/Cas9 editing (Fig. 4a, Supplementary Fig. 9b-d). Human $\mathrm{CD} 45^{+}$engraftment was observed at comparable high levels as in NSG mice (Fig. 4b). Strikingly, a threefold increase in CD41 ${ }^{+}$ 
a

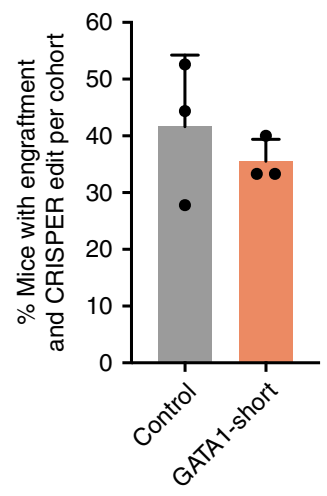

e

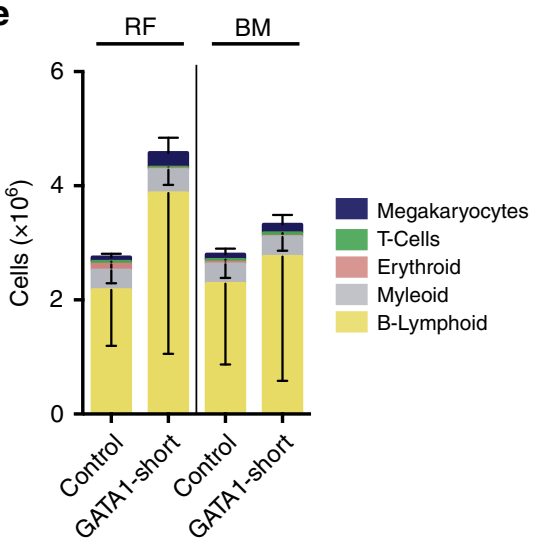

b

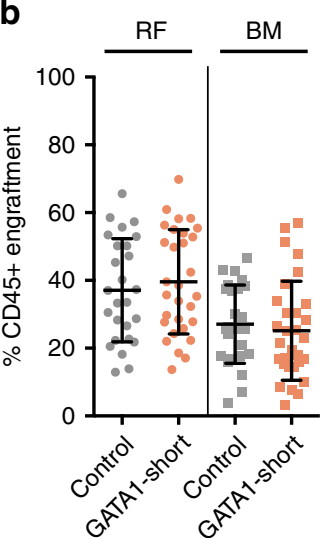

c

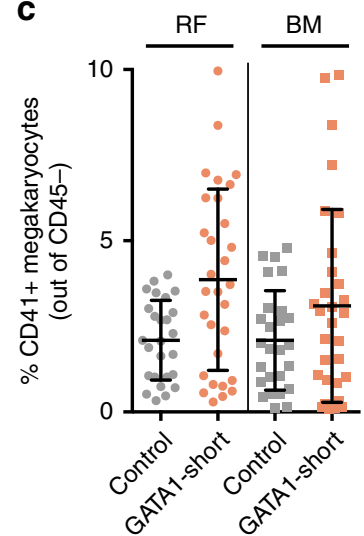

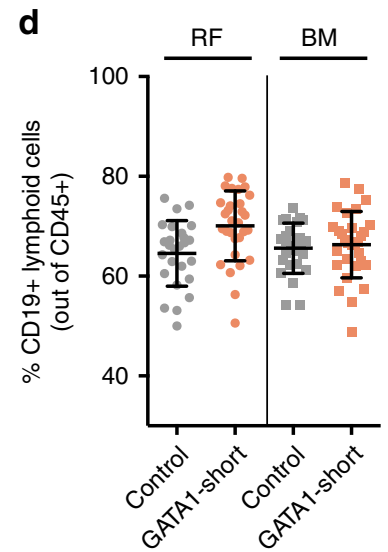

f
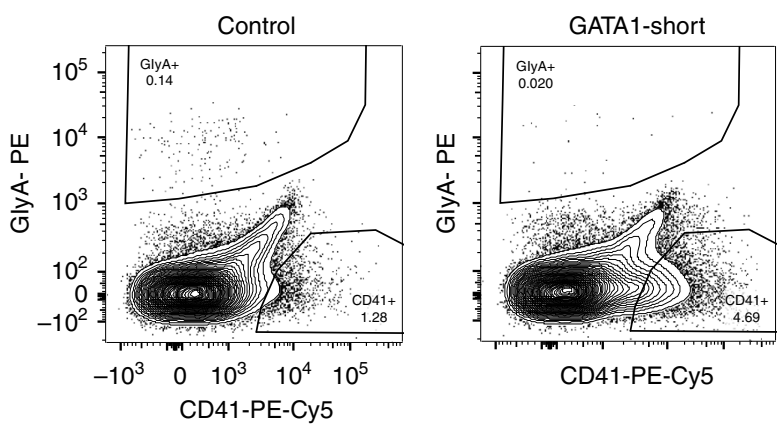

Fig. 3 Functional interrogation of single CRISPR/Cas9-edited hematopoietic stem cells in NSG mice. a Percentage of CRISPR/Cas9 edited LT-HSCs injected NSG mice with engraftment ( $>5 \%$ based on human $\mathrm{CD}_{4} 5^{+}$expression in RF) and high CRISPR/Cas9 knockout efficiency ( $>90 \%$ based on PCR and Sanger sequencing) from each of three cohorts ( $n=3$ animal cohorts with independent cord blood pools). $\mathbf{b}$ Engraftment levels of control and GATA1-short edited LT-HSCs injected NSG mice based on human CD45+ expression in RF and BM. c Percentage of CD41+CD45- megakaryocytes of control and GATA1Short edited LT-HSCs injected NSG mice in RF and BM (unpaired $t$ test $P<0.005$ for RF GATA1-Short versus RF control and unpaired $t$ test $P=0.107$ for BM GATA1-Short versus BM control). d Percentage of CD19+CD45+ lymphoid cells in control and GATA1-Short edited LT-HSCs injected NSG mice in RF and BM (unpaired $t$ test $P<0.005$ for RF GATA1-Short versus RF control). e Absolute cell numbers of different lineages in control and GATA1-Short edited LT-HSCs injected NSG mice in RF and BM (unpaired $t$ test $P<0.01$ for B-lymphoid RF GATA1-Short versus RF control and unpaired $t$ test $P<0.005$ for megakaryocytes RF GATA1-Short versus RF control). f Representative flow cytometry plots of control and GATA1-Short edited LT-HSCs injected NSG mice in RF showing $\mathrm{CD} 41^{+} \mathrm{CD} 45^{-}$megakaryocytes and GlyA+CD45- erythroid cells. Error bars represent standard deviations

megakaryocytic lineage output was observed in the RF and BM of mice transplanted with GATA1-Short edited LT-HSCs (Fig. 4c). No changes were seen in the percentage of B-lymphoid cells, but a decrease in human $\mathrm{GlyA}^{+} \mathrm{CD} 45^{-}$erythroid cells was detected in mice transplanted with GATA1-Short edited LT-HSCs (Fig. 4d, Supplementary Fig. 10a, b). Analysis of total cell numbers revealed a similar pattern, including a threefold increase in the number of megakaryocytic lineage derived cells and, at the same time, a sixfold reduction in erythroid cell numbers (Fig. 4e, Supplementary Fig. 10c-g). The cellular morphology of GlyA ${ }^{+} \mathrm{CD} 45^{-}$cells revealed more immature forms of erythroid cells and fewer enucleated erythroblasts in the RF of GATA1-Short edited LT-HSCs injected NSGW41 mice compared to control (Supplementary Fig. 11a, b) ${ }^{39}$. No difference in morphology was observed in $\mathrm{CD}_{4} 1^{+}$megakaryoblast-like cells in the $\mathrm{BM}$ of GATA1-Short edited LT-HSCs injected NSGW41 mice compared to control (Supplementary Fig. 11c). Only bulk CD $41^{+}$cells could be sorted, regardless of $\mathrm{CD} 45$ staining, since sufficient numbers of $\mathrm{CD} 41^{+} \mathrm{CD} 45^{-}$megakaryocytes could not be detected after the freeze/thaw cycle of stored BM. Furthermore, there were no differences in B-lymphoid proliferation in grafts generated by control- and GATA1-Short edited LT-HSCs in NSGW41 mice, in contrast to our observations in NSG recipients, which was possibly due to the reduced lymphoid bias observed in NSGW41 mice. In summary, xenotransplantation into NSGW41 mice further augmented the megakaryocytic lineage output of GATA1Short edited LT-HSCs in vivo, with a concomitant decrease in erythroid cells (Fig. 4f).

\section{Discussion}

We demonstrate that distinct numbers of isolated LT-HSCs as well as more committed stem and progenitor cells can be edited with high efficiency using CRISPR/Cas9. Conventional CRISPR/ Cas9 approaches on bulk $\mathrm{CD} 34^{+}$populations yield a mixed population of edited and unedited cells, making functional characterization difficult. Our approach, which requires retrospective verification of the CRISPR/Cas9 edits, permits the functional interrogation of LT-HSCs at a single cell level. Alternative approaches utilize HDR-mediated stable integration of selectable markers that allow prospective enrichment of CRISPR/ Cas9 edited cells upon expression of the fluorescent marker ${ }^{10,11}$. The advantages of our method are that no exogenous sequences are introduced into the genomic DNA and all regulatory processes such as splicing and spatial control of promoters and 

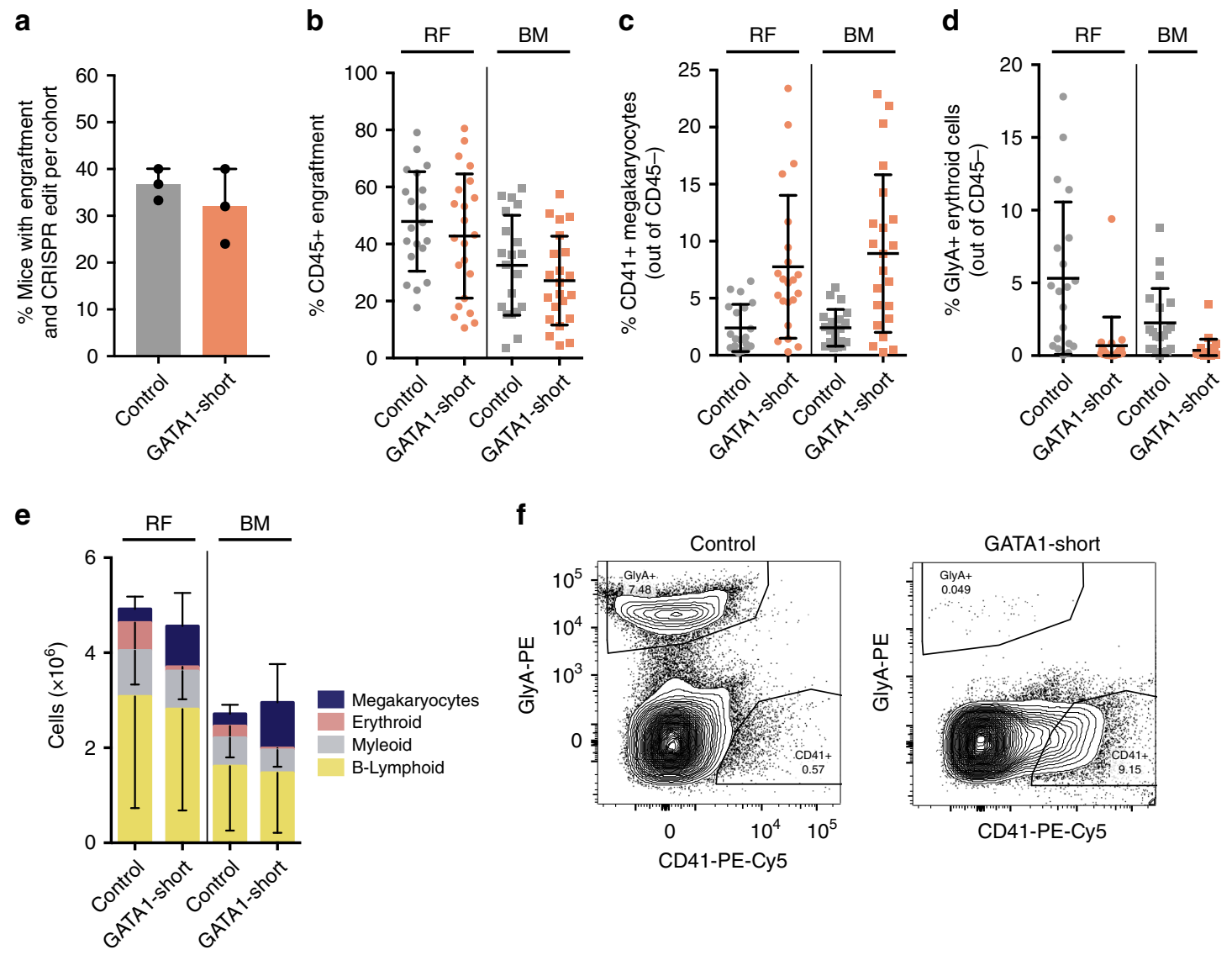

Fig. 4 Functional interrogation of single CRISPR/Cas9-edited hematopoietic stem cells in NSGW41 mice. a Percentage of CRISPR/Cas9 edited LT-HSCs injected NSGW41 mice with engraftment ( $>5 \%$ based on human CD45+ expression in RF) and high CRISPR/Cas9 knockout efficiency ( $>90 \%$ based on PCR and Sanger sequencing) from each of three cohorts ( $n=3$ animal cohorts with independent cord blood pools). $\mathbf{b}$ Engraftment levels of control and GATA1-short edited LT-HSCs injected NSGW41 mice based on human CD45 ${ }^{+}$expression in RF and BM. c Percentage of CD41 ${ }^{+}$CD45 ${ }^{-}$megakaryocytes of control and GATA1-Short edited LT-HSCs injected NSGW41 mice in RF and BM (unpaired t test $P<0.001$ for GATA1-Short versus control for both RF and BM). d Percentage of GlyA+D45- erythroid cells in control and GATA1-Short edited LT-HSCs injected NSGW41 mice in RF and BM (unpaired $t$ test $P<$ 0.0005 for RF GATA1-Short versus RF control and unpaired $t$ test $P<0.005$ for BM GATA1-Short versus BM control). e Total cell numbers of different lineages in control and GATA1-Short edited LT-HSCs injected NSGW41 mice in RF and BM (unpaired $t$ test $P<0.005$ for megakaryocytes GATA1-Short versus control for both RF and BM and unpaired $t$ test $P<0.005$ for erythroid GATA1-Short versus control for both RF and BM). $\mathbf{f}$ Representative flow cytometry plots of control and GATA1-Short edited LT-HSCs injected NSGW41 mice in RF showing CD41 ${ }^{+} \mathrm{CD} 45^{-}$megakaryocytes and GlyA ${ }^{+} \mathrm{CD} 45^{-}$ erythroid cells. Error bars represent standard deviations

enhancers are kept intact. In addition, we show for the first time that GATA1-Short isoform expression in LT-HSCs under the endogenous promoter leads to an increase in megakaryocytic output; this was not previously observed in cord blood derived bulk $\mathrm{CD} 4^{+} \mathrm{HSPCs}^{31}$. Aside from showing how a prototypical transcription factor functions in regulating lineage fate in LTHSCs, this result is of great importance in modeling the normal functions of the GATA1 transcription factor and the hematological diseases where it is mutated.

Interestingly, our in vitro and in vivo data seem to closely phenocopy several murine studies of GATA1 loss ${ }^{40}$, where megakaryocytes fail to undergo terminal differentiation and these immature megakaryocytes expand dramatically in the BM and spleen. Loss of GATA1 in mouse embryo-derived stem cells results in a maturation arrest at the proerythroblast stage of definitive erythroid precursors ${ }^{41}$ and ablation of GATA1 in adult mice results in a maturation arrest at the proerythroblast stage ${ }^{42}$. However, direct comparison of murine and human data is complicated by the fact that human and mouse GATA1-Short are normally produced by divergent mechanisms, where human GATA1-Short is generated through differential splicing and murine GATA1-Short is produced by alternative translation of a single $\mathrm{mRNA}^{43}$. Indeed,
GATA1-Short mRNA transcripts have not been reported in murine tissues $^{43}$. Furthermore, although fetal hematopoiesis is perturbed in mice with mutations conferring GATA1-Short expression, adult mice display no obvious hematopoietic defects ${ }^{32}$.

Children born with Down syndrome have an increased risk of developing AMKL, which is preceded by a pre-leukaemic syndrome termed transient leukemia that is characterized by high numbers of abnormal megakaryoblasts in the circulation, spleen and liver ${ }^{44}$. Nearly all cases of transient leukemia and Down syndrome associated AMKL have N-terminal truncating GATA1 mutations (GATA1-Short) present at birth that become undetectable after transient leukemia and AMKL remission $28,45,46$. Notably, several transgenic mouse models of trisomy 21 and GATA1-Short have been generated, yet none fully recapitulate the hematological abnormalities and malignancies seen in human trisomy $21^{32,47-49}$. Thus, the establishment of humanized in vivo model systems suitable to study the pathogenesis of Down syndrome blood malignancy is imperative. While our work described here represents an initial step, we foresee CRISPR/Cas9-mediated GATA1-Short editing in primary trisomy 21 LT-HSCs in combination with near clonal xenotransplantation as the most suitable approach to generate these models. 
Our observed phenotype of increased megakaryopoiesis due to forced expression of GATA1-Short seems to be at odds with a subset of Diamond Blackfan anemia patients, which is a genetic disease that usually presents in infancy and is characterized by low red blood cell counts. Most patients with Diamond Blackfan anemia harbor mutations in genes coding for ribosomal subunits, the most common being RPS19, that lead to a selective impaired production of full-length GATA1 and GATA1-Short ${ }^{23,50,51}$. Germline GATA1 mutations have also recently been described in patients with Diamond Blackfan anemia ${ }^{22-24}$, which lead to almost exclusive GATA1-Short expression, but no observed increase in megakaryocytes. Thus, it is possible that in these particular cases, germline GATA1 mutations severely constrained fetal blood development inducing an unidentified compensatory mechanism to allow for a more normalized megakaryocytic development. This selective pressure is further supported by the fact that mothers of children with Diamond Blackfan anemia have increased numbers of miscarriages ${ }^{52}$.

In summary, our method opens up the possibility of studying gene function relationships not only in LT-HSCs, but also in other stem and progenitor cells to uncover cell type specific phenotypes. We believe that the continuous improvement of CRISPR/Cas9 editing efficiency, for example through chemically modified gRNAs $s^{53-55}$ or different Cas9 variants $s^{56,57}$, will further improve this approach. In the future, we envision that this method could potentially be adapted for cellular therapies.

\begin{abstract}
Methods
Cord blood lineage depletion. Human cord blood samples were obtained from Trillium and William Osler hospitals with informed consent in accordance to guidelines approved by University Health Network (UHN) Research Ethics Board. Cord blood samples were processed $24-48 \mathrm{~h}$ after birth. Male samples were exclusively utilized in this study because GATA1 and STAG2 are located on the Xchromosome. This aided the CRISPR/Cas9 efficiency because of the need to edit only one X-chromosome. Control OR2W5 is located on chromosome 1. Samples were diluted 1:1 with phosphate-buffered saline (PBS) and mononuclear cells were enriched using lymphocyte separation medium (Wisent, 305-010-CL). Subsequently, red blood cells were lysed using an ammonium chloride solution (StemCell Technologies, 07850). Then, lineage positive cells were depleted by negative selection with the StemSep Human Hematopoietic Progenitor Cell Enrichment Kit (StemCell Technologies, 14056) and Anti-Human CD41 TAC (StemCell Technologies, 14050) according to the manufacturer's protocol. Lineage depleted cells were stored in 50\% PBS, 40\% fetal bovine serum (FBS) (ThermoFisher, 12483-020) and $10 \%$ DMSO (FisherScientific, D128-500) at $-150^{\circ} \mathrm{C}$.
\end{abstract}

Cord blood sorting. Lineage depleted cells were thawed via slow dropwise addition of X-VIVO 10 (Lonza, 04743Q) with 50\% FBS (Sigma, 15A085) and DNaseI (200 $\mu \mathrm{g} / \mathrm{ml}$, Roche, 10104159001). Cells were spun at $350 \times g$ for $10 \mathrm{~min}$ at $4{ }^{\circ} \mathrm{C}$ and then resuspended in PBS $+2.5 \%$ FBS. For all in vitro and in vivo experiments, the full stem and progenitor hierarchy sort as described in Notta et al. ${ }^{34}$ was utilized in order to sort LTHSCs, ST-HSCs, and MEPs. Lineage depleted cells were resuspended in $100 \mu \mathrm{l}$ per $1 \times$ $10^{6}$ cells and stained in two subsequent rounds for $20 \mathrm{~min}$ at room temperature each. First, the following antibodies were used (volume per $1 \times 10^{6}$ cells, all from BD Biosciences, unless stated otherwise): CD45RA FITC ( $5 \mu \mathrm{l}, 555488$, HI100), CD49f PE-Cy5

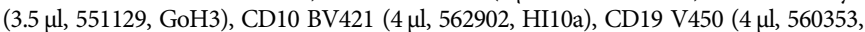
HIB19), and FLT3 CD135 biotin (12 $\mu$ l, clone 4G8, custom conjugation). After washing the cells, a second set of antibodies was used (volume per $1 \times 10^{6}$ cells, all from BD Biosciences, unless stated otherwise): CD45 V500 (4 $\mu \mathrm{l}, 560777$, HI30), CD34 APC-Cy7 ( $3 \mu \mathrm{l}$, clone 581, custom conjugation), CD38 PE-Cy7 ( $2.5 \mu \mathrm{l}, 335825, \mathrm{HB} 7)$, CD90 APC (4 $\mu \mathrm{l}, 559869,5 \mathrm{E} 10)$, CD7 A700 (10 $\mu \mathrm{l}, 561603, \mathrm{M}-\mathrm{T} 701)$, and Streptavidin Conjugate Qdot 605 (3 $\mu$ l, ThermoFisher, Q10101MP). Cell sorting was performed on the FACSAria III (BD Biosciences). LT-HSCs were sorted as $\mathrm{CD}^{-} 5^{+} \mathrm{CD} 34^{+} \mathrm{CD} 38^{-} \mathrm{CD} 45 \mathrm{RA}^{-}$ $\mathrm{CD} 0^{+} \mathrm{CD} 49 \mathrm{f}^{+}$, ST-HSCs as $\mathrm{CD} 45^{+} \mathrm{CD} 34^{+} \mathrm{CD} 38^{-} \mathrm{CD} 45 \mathrm{RA}^{-} \mathrm{CD} 90^{-} \mathrm{CD}^{-} \mathrm{f}^{-}$and MEPs as $\mathrm{CD}_{4}{ }^{+} \mathrm{CD} 34^{+} \mathrm{CD} 38^{+} \mathrm{CD} 10 / 19^{-} \mathrm{CD}^{-}{ }^{-} \mathrm{CD} 45 \mathrm{RA}^{-}{ }^{-} \mathrm{FLT}^{-}{ }^{-}$(Supplementary Figs. 1 and 2).

Pre-electroporation culture of sorted cells. Sorted LT-HSCs, ST-HSCs or MEPs were cultured for $36-48 \mathrm{~h}$ in serum-free X-VIVO 10 (Lonza) media with $1 \%$ Bovine Serum Albumin Fraction V (Roche, 10735086001), 1× L-Glutamine (Thermo Fisher, 25030081), 1× Penicillin-Streptomycin (Thermo Fisher, 15140122) and the following cytokines (all from Miltenyi Biotec): FLT3L (100 ng/ $\mathrm{mL})$, G-CSF $(10 \mathrm{ng} / \mathrm{mL})$, SCF $(100 \mathrm{ng} / \mathrm{mL})$, TPO $(15 \mathrm{ng} / \mathrm{mL})$, and IL-6 $(10 \mathrm{ng} / \mathrm{mL})$. Cells were cultured in 96-well U-bottom plates (Corning, 351177).
gRNA and HDR template design. gRNAs for GATA1 Short and Long were designed on Benchling (http://www.benchling.com). For GATA1 Short, gRNAs sequences were considered that were flanking the $5^{\prime}$ and $3^{\prime}$ end of exon 2 . Individual gRNAs targeting the $5^{\prime}$ or $3^{\prime}$ end were individually tested for cleavage efficiency and the best gRNA targeting each end was selected. Combined use of both gRNAs enabled complete excision of exon 2 (Fig. 1b). For GATA1 Long, gRNA sequences closest to the second ATG start codon were individually tested for cleavage efficiency and the best gRNA was selected. The GATA1 Long HDR template was designed with $60 \mathrm{bp}$ homology ends at either side. For the template, the ATG (Methionine) start codon was mutated to CTC (Leucine) and the PAM sequence was mutated from GGG (Glycine) to GGC (Glycine) in order to avoid repeated cutting by the gRNA (Fig. 1c). The control gRNAs, which target exon 1 of the olfactory receptor OR2W5, were predicted by the CRoatan algrotihm ${ }^{33}$. The STAG2 gRNA was predicted with the same algorithm.

gRNA and HDR template sequences:

Control gRNA-1: GACAACCAGGAGGACGCACT

Control gRNA-2: CTCCCGGTGTGGACGTCGCA

GATA1 Short gRNA-1: TGGAACGGGGAGATGCAGGA

GATA1 Short gRNA-2: CCACTCAATGGAGTTACCTG

GATA1 Long gRNA: CATTGCTCAACTGTATGGAG

GATA1 Long HDR template:

TCTTTCCTCCATCCCTACCTGCCCCCAACAGTCTTTCAGGTGTACCCA TTGCTCAACTGTCTCGAGGGCATCCCAGGGGGCTCACCATATGCCGGCT GGGCCTACGGCAAGACGGGGCTCTACCCTGCC

STAG2 gRNA: AATGGTCATCACCAACAGAA

CRISPR/Cas9 RNP electroporation. gRNAs were synthesized from IDT as Alt-R CRISPR/Cas9 crRNA, which require annealing with Alt-R tracrRNA (IDT) to form a functional gRNA duplex. The HDR template was synthesized from IDT as a single-strand Ultramer. crRNAs and tracrRNAs were resuspended to $200 \mu \mathrm{M}$ with TE Buffer (IDT). Both RNA oligonucleotides were mixed 1:1 to a final concentration of $100 \mu \mathrm{M}$ and annealed at $95^{\circ} \mathrm{C}$ for $5 \mathrm{~min}$ in a thermocycler, then cooled down to room temperature on the bench top. If using two gRNAs at the same time, both crRNAs were annealed to the tracrRNA in a single tube. For each reaction, $1.2 \mu \mathrm{l}$ crRNA:tracrRNA, $1.7 \mu$ l Cas9 protein (IDT) and $2.1 \mu \mathrm{l}$ PBS were combined in a low-binding Eppendorf tube (Axygen, MCT-175-C-S) and incubated for $15 \mathrm{~min}$ at room temperature. Subsequently, $1 \mu \mathrm{l}$ of $100 \mu \mathrm{M}$ electroporation enhancer (IDT) was added. Pre-electroporation cultured cells were washed in warm PBS and spun down at $350 \times g$ for $10 \mathrm{~min}$ at room temperature. Between $1 \times$ $10^{4}-5 \times 10^{4}$ cells per condition were resuspended in $20 \mu$ of Buffer P3 (Lonza) per reaction and quickly added to the Eppendorf tube containing the Cas9 gRNA RNP complex. The mixture was briefly mixed by pipetting and then added to the electroporation chamber (Lonza, V4XP3032). Cells were electroporated with the program DZ-100 using the Lonza Nucleofector and, immediately afterwards, $180 \mu \mathrm{l}$ of pre-warmed X-VIVO 10 media (as described above) was added. Cells were recovered overnight in the incubator before their use in in vivo or single cell in vitro assays.

Setup of single cell in vitro assay. Single cell in vitro assays were performed according to Notta et al. ${ }^{34}$ with slight modifications. Briefly, 3 days prior to the single cell assay, Nunc 96-well flat bottom plates (Thermo Fisher, 167008) were coated with $50 \mu \mathrm{l}$ per well of $0.2 \%$ Gelatin (G1393, Sigma-Aldrich) using a multichannel pipette. The first and last column of each 96-well plate were not utilized and instead filled with $150 \mu \mathrm{l}$ PBS per well to support proper humidity within the plate. After $1 \mathrm{~h}$, the $0.2 \%$ Gelatin is removed and murine MS- 5 stroma cells ${ }^{58}$ were seeded at a density of 1500 cells per well in $100 \mu \mathrm{l}$ H5100 media (StemCell Technologies, 05150). One-day prior, the H5100 media was removed and replaced with $100 \mu \mathrm{l}$ of erythro-myeloid differentiation media. For this, StemPro-34 SFM media (Thermo Fisher, 10639011) is used with the supplied supplement, 1× LGlutamine (Thermo Fisher, 25030081), 1× Penicillin-Streptomycin (Thermo Fisher, 15140122), $10 \mu \mathrm{l}$ Human LDL (StemCell Technologies, 02698) for every $50 \mathrm{ml}$ media and the following cytokines (all from Miltenyi Biotec unless stated otherwise): FLT3L $(20 \mathrm{ng} / \mathrm{mL})$, GM-CSF $(20 \mathrm{ng} / \mathrm{mL})$, SCF $(100 \mathrm{ng} / \mathrm{mL})$, TPO $(100 \mathrm{ng} / \mathrm{mL})$, EPO $(3 \mathrm{ng} / \mathrm{mL}$, Eprex), IL-2 $(10 \mathrm{ng} / \mathrm{mL}), \mathrm{IL}-3(10 \mathrm{ng} / \mathrm{mL}), \mathrm{IL}-6$ ( $50 \mathrm{ng} / \mathrm{mL})$, IL-7 $(20 \mathrm{ng} / \mathrm{mL})$, and IL-11 $(50 \mathrm{ng} / \mathrm{mL})$. On the day of the experiment, electroporated cells were stained with 1:2000 Sytox Blue (ThermoFisher, S34857) in PBS $+2.5 \%$ FBS and viable single cells were sorted and deposited into the MS-5 seeded 96 well plate using the FACSAria II (BD Biosciences). Single cells were cultured for 16-17 days with an addition of $100 \mu \mathrm{l}$ erythro-myeloid differentiation media per well at day 8 .

Flow cytometry of single cell in vitro assay. Wells with hematopoietic cell content were marked 1 day prior and the total number of wells with colonies was used to calculate CRISPR/Cas9 and single cell colony efficiencies. On the day of analysis, $140 \mu \mathrm{l}$ of media was removed from each well with a multichannel pipette and the content was mixed well. Upon additional washing of the wells with PBS, the content in each well was transferred to a 96-well filter plate (8027, Pall) in order to remove stromal cells. For this, the filter plate was put on top of a 96-well U-bottom plate (Corning, 351177) and centrifuged at $300 \times g$ for $7 \mathrm{~min}$ at room 
temperature. After the cells were pelleted at the bottom of the U-bottom plate, the media was tossed by quickly inverting the U-bottom plate $(\sim 20 \mu \mathrm{l}$ of liquid per well remained). Then, $30 \mu \mathrm{l}$ of PBS $+2.5 \%$ FBS was added and mixed into each well with a multichannel pipette and $25 \mu \mathrm{l}$ (half) was transferred to a 96-well PCR plate (Eppendorf, 951020362) for subsequent genomic DNA extraction (the PCR plate was sealed and stored at $-80 \mathrm{C}$ ). After that, $25 \mu \mathrm{l}$ of antibody mix in PBS $+2.5 \%$ FBS was added to each well, containing $25 \mu$ lof hematopoietic cells, and stained for 45 min at $4{ }^{\circ} \mathrm{C}$. The following antibodies were used (all antibodies from BD Biosciences, unless stated otherwise): CD45 APC (1:100, 560777, HI30), CD34 APCCy7 (1:250, clone 581, custom conjugation), CD33 BV421 (1:50, Biolegend, 303416, WM53), CD71 FITC (1:100, 347513, L01.1), CD41 PE-Cy5 (1:200, Beckman Coulter, 6607116, P2) and GlyA PE (1:200, Beckman Coulter, IM2211U, KC16). Finally, $150 \mu \mathrm{l}$ of PBS $+2.5 \%$ FBS were added to each well with a multichannel pipette and the cells were analyzed on the FACSCelesta with a high throughput sampler (HTS, BD Biosciences). All flow cytometry quantification was performed in a blinded manner. Generally, greater than ten cells were required to call a positive lineage. Erythroid cells were defined as positive upon CD71 expression, with or without expression of GlyA.

Genotyping of single cell in vitro assay. The PCR plates containing $25 \mu \mathrm{l}$ of cells per well were thawed and a modified protocol of the Agencourt GenFind V2 (Beckman Coulter, A41499) was utilized to isolate genomic DNA. Wells with cell content were transferred to a new 96-well PCR plate (Eppendorf, 951020362) in order to utilize multichannel pipetting for each future step. Totally, $25 \mu \mathrm{l}$ of lysis buffer and $1.2 \mu \mathrm{l}$ of Proteinase K (Zymo Research, D3001220) were pipetted into each well. After $30 \mathrm{~min}$ at room temperature, $50 \mu \mathrm{l}$ of magnetic particles were added. After $5 \mathrm{~min}$, the PCR plate was placed on a magnetic stand (ThermoFisher, AM10027) for $10 \mathrm{~min}$. The supernatant was removed with a multichannel pipette and the plate was taken off the magnet. Totally, $200 \mu \mathrm{l}$ of wash buffer 1 were mixed into each well and the PCR plate was put back onto the magnetic stand. After 10 $\mathrm{min}$, the supernatant was removed and each well was washed with $125 \mu \mathrm{l}$ of wash buffer 2. Finally, after the last wash buffer was removed, the magnetic particles were resuspended with $60 \mu \mathrm{l}$ of TE buffer (IDT). The PCR plate was put back on the magnetic stand and after $10 \mathrm{~min}, 57 \mu \mathrm{l}$ of eluted genomic DNA was removed and put into a new PCR plate.

The CRISPR/Cas9 engineered genomic locus was amplified via PCR. For each PCR reaction, $23 \mu \mathrm{l}$ of eluted genomic DNA was mixed with $1 \mu \mathrm{l}$ of forward and reverse primer $(10 \mu \mathrm{M})$ and $25 \mu \mathrm{l}$ of AmpliTaq Gold 360 Master Mix

(ThermoFisher, 4398881 ). The PCR program was: $95^{\circ} \mathrm{C}$ for $10 \mathrm{~min}$, followed by 95 ${ }^{\circ} \mathrm{C}$ for $30 \mathrm{~s}, 56^{\circ} \mathrm{C}$ for $30 \mathrm{~s}$ and $72^{\circ} \mathrm{C}$ for $1 \mathrm{~min}\left(40\right.$ cycles) and then $72^{\circ} \mathrm{C}$ for $7 \mathrm{~min}$. To identify colonies with the GATA1-Short genotype, $15 \mu$ l of PCR product was run on a $1.5 \%$ agarose gel (ThermoFisher, 16500500). Control gRNA colonies were screened for homozygous deletion of OR2W5 (deletion within exon 1, 700 to 500 bp, (Supplementary Fig. 4a). Similarly, GATA1-Short colonies were screened for a shift in the size of the PCR product (deletion of exon 2, 1000 to $550 \mathrm{bp}$, Supplementary Fig. 4b). In order to identify colonies with the GATA1-Long genotype, PCR products were column purified using the ZR-96 DNA Clean-up Kit (Zymo Research, D4018) according to the manufacturer's protocol. The purified PCR product was Sanger sequenced using the reverse PCR primer and the chromatograms were inspected to identify colonies that contained the alternative start site mutation (Supplementary Fig. 4c). Finally, to identify STAG2 knock-out colonies, PCR products were column purified and sent for Sanger sequencing using the reverse PCR primer. Only colonies that showed a frame shift mutation were considered positive (Supplementary Fig. 6f).

PCR primers:

Control (OR2W5) forward primer: 5'-TCGGCCTGGACTGGAGAAAA-3' Control (OR2W5) reverse primer: 5'-GAGACCACTGTGAGGTGAGA-3' GATA1-Short forward primer: 5'-CAGGAGAGAATGAGAAAAGAGTGGA-3' GATA1-Short reverse primer: $5^{\prime}$-ATTTCCAAGTGGGTTTTTGAGGAT-3' GATA1-Long forward primer: 5'-GCCACACTGAGAGGCAATACT-3' GATA1-Long reverse primer: 5'-AAAAGTCAGGGCCCCCATAAG-3' STAG2-forward primer: 5'-CCACAAAGAGGCTGTCACAGTT-3'

STAG2-reverse primer: 5'-CATGCAGCAGAAAATGAATCAAAAC-3'

Western assay. MEPs were sorted and CRISPR/Cas9 RNP electroporated as described above. After electroporation, MEPs were cultured in erythro-myeloid differentiation media (as described above for single cells in vitro assays) for 3 days. $1 \times 10^{5}$ cells were then lysed in RIPA buffer (ThermoFisher, 899000) containing protease and phosphatase inhibitors (ThermoFisher, 78446). Subsequently, samples were centrifuged at $12,000 \mathrm{~g}$ for $5 \mathrm{~min}$ at $4{ }^{\circ} \mathrm{C}$ and supernatants were utilized for Western assay. Western assay was performed on the size-based Wes capillary platform (ProteinSimple) using the $12-230 \mathrm{kDa}$ capillary cartridge according to manufacturer's protocol. In order to determine the optimal antibody dilution, antiGATA-1 antibody (Cell Signaling D24E4) was titrated prior to use on a lysate from $\mathrm{CD}^{+} 4^{+}$cord blood and 1:5 dilution was used in the samples.

Animal studies. All mouse experiments were approved by the University Health Network (UHN) Animal Care Committee and we complied with all relevant ethical regulations for animal testing and research. All mouse transplants were performed

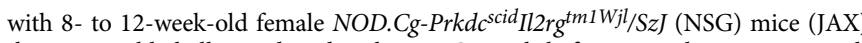
that were sublethally irradiated with $225 \mathrm{cGy}, 24 \mathrm{~h}$ before transplantation, or with 8- to 12-week-old female NOD.Cg-Prkdscid Il2rgtmlWjl Kitem1Mvw/SzJ (NSGW41) mice that were not irradiated. Sample size was chosen to give sufficient power for calling significance with standard statistical tests. Intrafemoral injections were performed as described in Mazurier et $\mathrm{al}^{59}$. For this, mice were anesthetized with isoflurane and the right knee was secured in a bent position to drill a hole into the RF with a 27 gauge needle. Then, 100-250 CRISPR/Cas9-edited LT-HSCs were injected in $30 \mu \mathrm{l}$ PBS using a 28 gauge $1 / 2$ cc syringe (Becton Dickinson, 329461). LT-HSC cell numbers are based on the number of flow cytometry sorted cells at day 0 . After 12 or 24 weeks, mice were sacrificed to obtain the RF and BM (left femur and both tibias, BM). Bones were flushed in $1 \mathrm{~mL}$ PBS $+2.5 \%$ FBS and cells were centrifuged at $350 \times \mathrm{g}$ for $10 \mathrm{~min}$. Cells were resuspended in $500 \mu \mathrm{l}$ of PBS + $2.5 \%$ FBS. Subsequently, cells from BM and RF were counted in ammonium chloride (StemCell Technologies, 07850) using the Vicell XR (Beckman Coulter). Totally, $25 \mu \mathrm{l}$ of cells were used for flow cytometry analysis and another $25 \mu \mathrm{l}$ were frozen down for genomic DNA isolation in order to verify CRISPR/Cas9 edits (same protocol as above for single cell in vitro assays).

Limiting dilution in vivo assays. For limiting dilution transplantation assays, CRISPR/Cas9 control gRNA electroporated LT-HSCs were injected at defined doses (equivalent to $25,50,100$, and 200 LT-HSCs) into 8- to 12 -week-old female NSG or NSGW41 mice. LT-HSC cell numbers were based on the number of flow cytometry sorted cells at day 0. LT-HSC frequency was estimated using the online tool ELDA (http://bioinf.wehi.edu.au/software/elda/index.html) ${ }^{36}$.

Flow cytometry of in vivo assays. Cells from the RF and BM of transplanted mice were stained in 96-well U-bottom plates (Corning, 351177) for $45 \mathrm{~min}$ at $4{ }^{\circ} \mathrm{C}$. The following antibodies were used (all from BD Biosciences, unless stated otherwise): CD45 APC-Cy7 (1:100, 348795560566), CD45 A700 (1:100, 560566), CD33 APC (1:100, 340474), and CD19 V450 (1:100, 560353), CD41 PE-Cy5 (1:200, Beckman Coulter, 6607116), GlyA PE (1:100, Beckman Coulter, IM2211U), and CD3 FITC (1:100, 349201). For NSGW41 mice at 12 weeks, CD3 FITC staining was omitted. Cells were analyzed on the FACSCelesta (BD Biosciences).

CRISPR/Cas9 efficiency in cells and engrafted mice. After each CRISPR/Cas9 RNP electroporation, a small subset of cells was cultured in X-VIVO 10 media (as described above) for 5-7 days in order to validate CRISPR/Cas9 efficiency. Genomic DNA was isolated from bulk cells and the CRISPR/Cas9 engineered genomic locus was amplified via PCR as described above. Sanger sequencing was carried out using the reverse PCR primer and the chromatograms were analyzed using the online tool TIDE (https://tide.deskgen.com/ $/)^{60}$ in order to verify CRISPR/ Cas9 editing in control, GATA1-Short and GATA1-Long edited bulk cells. Because of the large deletion size of control (200 bp) and GATA1-Short ( $400 \mathrm{bp}$ ) edited cells, the CRISPR/Cas9 efficiency was evaluated based on the percentage of aberrant sequences after the gRNA cut site (Supplementary Fig. 7e).

For each transplanted mouse, CRISPR/Cas9 efficiency of control and GATA1Short edited cells was evaluated in the RF using the same approach. Only mice that showed a CRISPR/Cas9 knockout efficiency of $>90 \%$ as determined by the percentage of aberrant sequences after the gRNA cut site and a CD $45^{+}$engraftment level in the RF of $>5 \%$ were utilized in the analysis. Because only one Xchromosome needed to be edited for GATA1-Short, single clonal engraftment was visible based on individual chromatograms (Supplementary Fig. 7f). GATA1-Short edited LT-HSCs transplanted mice with more than one clone were included into our near-clonal xenotransplantation analysis, as long as the CRISPR/Cas9 knockout efficiency of $>90 \%$ and engraftment criteria of $>5 \%$ were satisfied.

Methylcellulose colony formation assay. Totally, $1 \times 10^{5}$ cells from the RF of xenotransplanted mice were transferred to $1 \mathrm{ml}$ of MethoCult H4034 Optimum methylcellulose medium (StemCell Technologies, 04034) and plated onto a $35 \mathrm{~mm}$ dish for human-specific colony formation. After 10-11 days, individual colonies were collected, washed in PBS and genomic DNA was isolated as described above. Individual CRISPR/Cas9 edits were determined using PCR amplification and Sanger sequencing with the reverse PCR primer as described above.

Cellular morphology. The RF and BM of genetically verified control and GATA1Short edited LT-HSCs injected NSGW41 mice were thawed and five samples per condition were pooled together. Murine cells were depleted from each pooled sample using the Mouse Depletion Kit (Miltenyi Biotec). Cells were stained for 30 min at $4{ }^{\circ} \mathrm{C}$ with the following antibodies: CD45 V500 (1:100, 560777, HI30), GlyA PE (1:100, Beckman Coulter, IM2211U), and CD41 PE-Cy5 (1:200, Beckman Coulter, 6607116, P2). GlyA ${ }^{+} \mathrm{CD}_{45}{ }^{-}$sorted cells from RF were washed in PBS with 2.5\% FBS and then cytospinned using the CytoSpin 4 instrument (112×g for 10 min, high acceleration, ThermoFisher) according to manufacturer's instructions. $\mathrm{CD} 41^{+}$sorted cells from BM were cytospinned at $55 \times g$ for 10 min with medium acceleration. 
CRISPR/Cas9 off-target analysis. Genomic loci that were similar to the gRNA target sequence were identified with Cas0-OFFinder (http://www.rgenome.net/casoffinder $^{61}$, using a mismatch number of $2-4$ and a DNA/RNA bulge size of 0 . Two genomic loci with 2 mismatches and 10 genomic loci with 3 mismatches were chosen for GATA1-Short gRNA-1, 8 genomic loci with 3 mismatches and 1 genomic loci with 4 mismatches for GATA1-Short gRNA-2, 4 genomic loci with 3 mismatches and 7 genomic loci with 4 mismatches for GATA1-Long gRNA-1, 3 genomic loci with 2 mismatches, 2 genomic loci with 3 mismatches and 6 genomic loci with 4 mismatches for Control gRNA-1, and 1 genomic loci with 3 mismatches and 11 genomic loci with 4 mismatches for Control gRNA-2. PCR primers were designed to amplify $500 \mathrm{bp}$ around these genomic loci. 20-30 single cell colonies that were positively identified with the correct CRISPR/Cas9 edit were selected from each cell type and genotyped for PCR amplification. PCR products were column purified and subsequent Sanger sequencing with both the forward and reverse PCR primer was carried out. TIDE analysis was used to assess any CRISPR/Cas9 cleavage efficiency.

PCR primers:

FW_GATA1Short_gRNA1_chr4_118059061: 5' -

CCCTCAAACTTCCCGACTAAGG-3

RV_GATA1Short_gRNA1_chr4_118059061: 5' GCTCAGATGTGACTGGGCG-3

FW_GATA1Short_gRNA1_chr16_35544187: 5' CGCAGGTTTAGCAAAGTTCCC-3'

RV_GATA1Short_gRNA1_chr16_35544187: 5'-

CCAGATGGGTTTCTGCTTTTACGA-3'

FW_GATA1Short_gRNA1_chr22_44756609: 5' -

GGGTTTTTCTGGGCTGCAAG-3'

RV_GATA1Short_gRNA1_chr22_44756609: 5' -

ACATAATAGTATTGCAGTAATGCGG-3'

FW_GATA1Short_gRNA1_chr19_32673314: 5' -

CTCTAGGGCCGCTTCTCAGT-3'

RV_GATA1Short_gRNA1_chr19_32673314: 5'-

ACGCTACATAGGGTCGTTCC-3'

FW_GATA1Short_gRNA1_chr15_52109094: 5' -

GTTTGGACTGGTCGAGCCT-3'

RV_GATA1Short_gRNA1_chr15_52109094: 5' -

GAGTGCGAACCTCTCATCTCTC-3

FW_GATA1Short_gRNA1_chr10_53712490: 5' -

CCAGTCAAGCACCTAGCGTA-3'

RV_GATA1Short_gRNA1_chr10_53712490: 5' -

GACTAGAAAGGCCTGAACCTCT-3'

FW_GATA1Short_gRNA1_chr14_72742463: 5' -

GGTCCCTTCCAGCACGTAAT-3'

RV_GATA1Short_gRNA1_chr14_72742463: 5' -

CCTCCCAATCCGGGAACAAC-3'

FW_GATA1Short_gRNA1_chr16_71816748: 5' -

TCTGGGGTACTAGAGGGGAAC-3

RV_GATA1Short_gRNA1_chr16_71816748: 5' -

TCAGGCCAGCATACATCGTG-3'

FW_GATA1Short_gRNA1_chr1_223704955: 5' -

CTGAAATGTCGGCCCTGCAA-3'

RV_GATA1Short_gRNA1_chr1_223704955: 5'

CCGAGGATGTAGTCATGGTGG-3'

FW_GATA1Short_gRNA1_chr17_80332977: 5' -

CCTTGGGTCTGCGTGAAGAT-3'

RV_GATA1Short_gRNA1_chr17_80332977: 5' -

AGTCACCTCTTAACTCTAACAGAAA-3'

FW_GATA1Short_gRNA1_chr10_20221643: 5' -

AGTGCAGTTAGAACAGACCAGC-3'

RV_GATA1Short_gRNA1_chr10_20221643: 5' -

GACAGACCTGTCAATCTGCTTTAT-3'

FW_GATA1Short_gRNA1_chr18_8376641: 5' -

GAGGACTGGCTGTTTCATCG-3'

RV_GATA1Short_gRNA1_chr18_8376641: 5'

CACTATTCTCAGGAGCGGTCT-3'

FW_GATA1Short gRNA2 chr5_178537945: 5'-

CATGACAGGCGCCTTCTTTACT-3'

RV_GATA1Short_gRNA2_chr5_178537945: 5' -

GAACATCTCCCCTTCAGCTCCT-3'

FW_GATA1Short_gRNA2_chr13_113094529: 5' -

TAGGTGTTGAACATGACCGTGG-3'

RV_GATA1Short_gRNA2_chr13_113094529: 5'

ACAAAGTGCTGCTGAGACAGAG-3'

FW GATA1Short gRNA2_chr21 42868084: 5'-

AGCACACAACCTTCAAAAGACG-3'

RV GATA1Short gRNA2 chr21 42868084: 5'-

GTGGGTGGAATGTCACCTTACT-3'

FW_GATA1Short_gRNA2_chr10_123397080: 5'

AATGCTGAGAGGCTTCCATTCC-3'

RV_GATA1Short_gRNA2_chr10_123397080: 5' -

CAAGATGTGCCTCCTCTCTGTG-3
FW_GATA1Short_gRNA2_chr8_35663175: 5' TCACAAGTTTGTTAGAGCGGTG-3'

RV_GATA1Short_gRNA2_chr8_35663175: 5' -

TGCTCTCAGCAGTAGATCTGG-3'

FW_GATA1Short_gRNA2_chr8_107174119: 5' -

CTCAGCGAGGCACAGAATTG-3'

RV_GATA1Short_gRNA2_chr8_107174119: 5' -

AGCGGCTACCCCATAATAGC-3'

FW_GATA1Short_gRNA2_chr12_2085541: 5' -

TTAAGGCAATGTCAACCTTTTCCA-3

RV_GATA1Short_gRNA2_chr12_2085541: 5' -

AGACGATGCAGGACCACCTA-3'

FW_GATA1Short_gRNA2_chr3_127784219: 5' -

TAACCCCCTTTGGGAGCACA-3'

RV_GATA1Short_gRNA2_chr3_127784219: 5' -

GCAGTGGGACGGCTATATCT-3'

FW_GATA1Short_gRNA2_chr7_17679912: 5' -

AGTATGCACATCCAGTTGGGG-3'

RV_GATA1Short_gRNA2_chr7_17679912: 5' -

CTGCTTGCACTGCTTAACGA-3'

FW_GATA1Long_gRNA_chr8_109403836: 5' -

CCCAAATATCAGTTCCATTGGGC-3'

RV_GATA1Long_gRNA_chr8_109403836: 5'-

GCAGCTGTTAACAAGAGAGTGC-3'

FW_GATA1Long_gRNA_chr3_77895170: 5' -

TTCCTCGCATCACATACGCC-3' RV_GATA1Long_gRNA_chr3_77895170: 5' -

ACCAGATTTCAGCCACTTGGA-3'

FW_GATA1Long_gRNA_chr4_22175316: 5'

AGCGCTTGAATACCTTCAGCAT-3'

RV_GATA1Long_gRNA_chr4 22175316: 5' -

GGAGGTGGCACAAGGTAGGTA-3'

FW_GATA1Long_gRNA_chr13_51458879: 5' -

AGAATGGTGACCCTGGCTTA-3

RV_GATA1Long_gRNA_chr13_51458879: 5'-ATTGGCTGGACAGTTTCGC-3' FW GATA1Long gRNA chr15 62326516: 5'-

TAGGCGGGAATGTCAAAGCC-3' RV_GATA1Long_gRNA_chr15_62326516: 5' -

GAGCTGGGACCCACGATAAAA-3' FW_GATA1Long_gRNA_chr8_81290900: 5'

AACGGGCACTTTAACAGGGAA-3'

RV_GATA1Long_gRNA_chr8_81290900: 5' -

AACCTTTGTCAGACCAACTCTGT-3'

FW GATA1Long gRNA chr7 40859472: 5'

AAGCTCGTTTGCTGCTTTACTG- 3' $^{\prime}$

RV_GATA1Long_gRNA_chr7_40859472: 5' -

CTCCGCTCTTATGCTCTCTAGC-3'

FW_GATA1Long_gRNA_chr12_20017977: 5' -

ATTAGGTCAGTCCAAGCCAACA-3

RV_GATA1Long_gRNA_chr12_20017977: 5'

TCTCССТCTTATCAATACCCCCAAC-3'

FW GATA1Long gRNA chr12 94844478: 5' -

TTGCTAGATACAGTCCTTGCCTG-3'

RV GATA1Long_gRNA_chr12_94844478: 5'

CCATTGTCTTGCAGATGTTCTCA-3'

FW_GATA1Long_gRNA_chr2_15662510: 5' -

ACCGACATGGGCCTAAGGATA-3' RV_GATA1Long_gRNA_chr2_15662510: 5' -

GGCAGCTTGTCTTGAGAATGG-3'

FW GATA1Long gRNA chr9 76619884: 5' -

ATCCCTCTCAAGCAGCCTAAT-3

RV GATA1Long gRNA chr9 76619884: 5' -

ATTTGCTCACAGAGAAATCACACCA-3 FW_Control_gRNA1_chr7_3081168: 5'

AAATTATCCACATTAATCGGGAAGCC-3' RV_Control_gRNA1_chr7_3081168: 5'

TTGTTTCAAAATATCACCGTTGGCA-3 FW_Control_gRNA1_chr7_135691300: 5' -

CTTTGAGTCTTTCCTCCCATGC-3 RV_Control_gRNA1_chr7_135691300: 5'

ACTGGGGGTAGCTCTAGTTGAA-3' FW_Control gRNA1_chr3 55088990: 5'-CTGTGTCACTTGGCTCGTCT-3' RV Control gRNA1 chr3 55088990: 5'-CATGTAGTGAGTACCGGGGC-3' FW_Control_gRNA1_chr5_4882788: 5'

AGCAGCCAACTTGGAAACATAAG-3'

RV_Control_gRNA1_chr5_4882788: 5'-AATCAGGCCTCAGAACCATCTT-3' FW Control gRNA 1 chr16 48579049: 5' -

TTACCACACTCCACTCCTCTGT-3' RV_Control_gRNA1_chr16_48579049: 5'

CCTGTGTTCCTGAACCTCCTAAT-3' 
FW_Control_gRNA1_chr1_191051674: 5'ATCCAAGCATACATGACAGAGAT-3' RV_Control_gRNA1_chr1_191051674: 5' GTATCTTGTGGGCACAACCTTT-3' FW Control gRNA1_chr12 114950867: 5' CAATCCTGCCACATTTCTGCATAC-3' RV_Control_gRNA1_chr12_114950867: 5' CTTGCCTGTCTCTCAAAGCATC-3' FW_Control_gRNA1_chr21_34622361: 5' AGGATTTCAGCTCTAGCAACCA-3 RV_Control_gRNA1_chr21_34622361: 5' CCCCATTTTAGACTTATGCCCCC-3' FW_Control_gRNA1_chr2_30448683: 5'-

CGTAATAGCGCTTGTGTTTCAGT-3' RV Control gRNA1_chr2 30448683: 5'-GCTAGATGACTGAGTTGGCAC-3' FW_Control_gRNA1_chr6_169886852: 5' -

AACAGATGATTCTGCAGGCAAAC-3' RV_Control_gRNA1_chr6_169886852: 5'-

GACGATCTTTTAGCTGTCCCCA-3' FW_Control_gRNA1_chr20_52425093: 5'-

CAACCTGTACTCTGGGACGTAG-3 RV_Control_gRNA1_chr20_52425093: 5'-

CGTGGAAACTTCTTCCTTGTCG-3

FW_Control_gRNA2_chr4_1100146: 5'-TGTAAAGGCGTGCTGTTGGT-3' RV_Control_gRNA2_chr4_1100146: 5'-ATCTGACGGTGTTGTGACTGG-3' FW_Control_gRNA2_chr16_85653916: 5'-GTGTGTGTGAAGGCCGGTA-3' RV_Control_gRNA2_chr16_85653916: 5'-CTCCACAATGAGGCGTTCCC-3' FW Control gRNA2 chr2 118858930: 5'-CAGGAGACGCTCCAAGTCG-3' RV_Control_gRNA2_chr2_118858930: 5'-TCCCTCACGTACCAGTCGG-3' FW_Control_gRNA2_chr21_42232307: 5'-ATGGACCAACTCTCACAGCA-3' RV_Control_gRNA2_chr21_42232307: 5'-GGCCTCCATTTGAGAACGTG-3' FW_Control_gRNA2_chr12_12496109: 5'-

TGTGGCGTGTAGGTAAAGAGAC-3'

RV_Control_gRNA2_chr12_12496109: 5' -

TGTATCTGAGGTGAACCACTGC-3'

FW Control gRNA2 chr19 18226209: 5'-

GACACCCAGAGTTCATTGTGAG-3

RV_Control_gRNA2_chr19_18226209: 5'-

GCTTTTGGTGAATCAGAGCATCC-3' FW_Control_gRNA2_chr14_96046026: 5' -

CATGGTGTGGACTGTTTCTGTT-3

RV_Control_gRNA2_chr14_96046026: 5'-

AGGTTTTGCCAAGCTCTACACA-3'

FW Control gRNA2 chr5 2514714: 5' -CACAGGGTTTGGTGTTTGTAGG-3' RV_Control_gRNA2_chr5_2514714: 5'-TGATCTGGCTTGACTTGCTTCC-3' FW_Control_gRNA2_chr8_10811909: 5'

AGGAAGACAAATCCTAAGACAGCC-3 RV_Control_gRNA2_chr8_10811909: 5' -

AAGTTTTACGTTTTCCTCGACCAC-3'

FW_Control_gRNA2_chr3_122079794: 5' -

AGATAAGCCAAGCCAGATTCTTCA-3'

RV Control gRNA2 chr3 122079794: 5' -

CTGCTTGGAATTCCCACATTACTC-3'

FW_Control_gRNA2_chr7_2644811: 5'-

GTTTATAGACAACCCACTGAGCAC-3'

RV_Control_gRNA2_chr7_2644811: 5'-

CTCATTGGCACTACTGCAGAGAAA-3'

FW_Control_gRNA2_chr15_54082949: 5' -

ACTCATCTTAAGGAACCGTCGC-3

RV_Control_gRNA2_chr15_54082949: 5'

CATAGCCACATCCAAAGGTCCA-3'

Karyotyping analysis. Lineage depleted cord blood cells were thawed and CD $34^{+}$ $\mathrm{CD} 38^{-}$cells were sorted using CD34 APC-Cy7 (clone 581, custom conjugation) and CD38 PE-Cy7 (335825, HB7). Cells were pre-cultured for $36-48 \mathrm{~h}$ in serum-free media and subsequently electroporated as described above. After overnight incubation, media was changed to IMDM (Thermo Fisher, 12440061) with 10\% FBS (Sigma, 15A085), 1× L-Glutamine (Thermo Fisher, 25030081), 1× Penicillin-Streptomycin (Thermo Fisher, 15140122) and the following cytokines (all from Miltenyi Biotec): FLT3L (100 ng/mL), G-CSF (10 ng/mL), SCF (100 ng/mL), TPO (15 ng/mL), and IL-6 $(10 \mathrm{ng} / \mathrm{mL})$ to allow for cell expansion. Subsequently, karyotyping of chromosomes was performed according to standard procedures. Metaphase slides were prepared, then banded with trypsin and stained with Leishman's stain. The G-banded slides were scanned and metaphases captured using an automated imaging system (MetaSystems). Metaphases were analyzed using Ikaros image analysis software (MetaSystems). For each condition, 20 metaphases were analyzed by G-banded karyotyping for numerical and structural abnormalities.

Statistical analysis. Error bars represent standard deviations. Statistical significance was assessed using two-tailed unpaired student's $t$ test.
Life sciences reporting summary. Additional information on experimental design is available in the Nature Research Reporting Summary linked to this article.

Reporting summary. Further information on research design is available in the Nature Research Reporting Summary linked to this article.

\section{Data availability}

All datasets generated in this study are available within the paper or from the corresponding author upon reasonable request. Full length gel pictures and western assay can be found in Supplementary Fig. 12

Received: 5 April 2019; Accepted: 29 September 2019; Published online: 18 October 2019

\section{References}

1. Laurenti, E. et al. CDK6 levels regulate quiescence exit in human hematopoietic stem cells. Cell Stem Cell 16, 302-313 (2015).

2. Notta, F. et al. Isolation of single human hematopoietic stem cells capable of long-term multilineage engraftment. Science 333, 218-221 (2011).

3. Porteus, M. H. A new class of medicines through DNA Editing. N. Engl. J Med. 380, 947-959 (2019).

4. Dever, D. P. \& Porteus, M. H. The changing landscape of gene editing in hematopoietic stem cells: a step towards Cas9 clinical translation. Curr. Opin. Hematol. 24, 481-488 (2017).

5. Shlush, L. I. et al. Identification of pre-leukaemic haematopoietic stem cells in acute leukaemia. Nature 506, 328-333 (2014).

6. Corces-Zimmerman, M. R., Hong, W.-J., Weissman, I. L., Medeiros, B. C. \& Majeti, R. Preleukemic mutations in human acute myeloid leukemia affect epigenetic regulators and persist in remission. Proc. Natl Acad. Sci. USA 111, 2548-2553 (2014).

7. Shlush, L. I. et al. Tracing the origins of relapse in acute myeloid leukaemia to stem cells. Nature 547, 104-108 (2017).

8. Tothova, Z. et al. Multiplex CRISPR/Cas9-based genome editing in human hematopoietic stem cells models clonal hematopoiesis and myeloid neoplasia. Cell Stem Cell 21, 547-555.e8 (2017).

9. Schneidawind, C. et al. MLL leukemia induction by $\mathrm{t}(9 ; 11)$ chromosomal translocation in human hematopoietic stem cells using genome editing. Blood Adv. 2, 832-845 (2018)

10. Bak, R. O., Dever, D. P. \& Porteus, M. H. CRISPR/Cas9 genome editing in human hematopoietic stem cells. Nat. Protoc. 13, 358-376 (2018)

11. Bak, R. O. et al. Multiplexed genetic engineering of human hematopoietic stem and progenitor cells using CRISPR/Cas9 and AAV6. eLife 6, 18 (2017)

12. Brunetti, L., Gundry, M. C., Kitano, A., Nakada, D. \& Goodell, M. A. Highly efficient gene disruption of murine and human hematopoietic progenitor cells by CRISPR/Cas9. J. Vis. Exp. https://doi.org/10.3791/57278 (2018)

13. Gundry, M. C. et al. Highly efficient genome editing of murine and human hematopoietic progenitor cells by CRISPR/Cas9. Cell Rep. 17, 1453-1461 (2016)

14. Lattanzi, A. et al. Optimization of CRISPR/Cas9 delivery to human hematopoietic stem and progenitor cells for therapeutic genomic rearrangements. Mol. Ther. 27, 137-150 (2019).

15. Mandal, P. K. et al. Efficient ablation of genes in human hematopoietic stem and effector cells using CRISPR/Cas9. Cell Stem Cell 15, 643-652 (2014).

16. Dever, D. P. et al. CRISPR/Cas9 $\beta$-globin gene targeting in human haematopoietic stem cells. Nature 539, 384-389 (2016).

17. Wu, Y. et al. Highly efficient therapeutic gene editing of human hematopoietic stem cells. Nat. Med. 387, 2554 (2019).

18. Schiroli, G. et al. Precise gene editing preserves hematopoietic stem cell function following transient p53-mediated DNA damage response. Cell Stem Cell 24, 551-565.e8 (2019).

19. Leonard, M., Brice, M., Engel, J. D. \& Papayannopoulou, T. Dynamics of GATA transcription factor expression during erythroid differentiation. Blood 82, 1071-1079 (1993).

20. Martin, D. I., Zon, L. I., Mutter, G. \& Orkin, S. H. Expression of an erythroid transcription factor in megakaryocytic and mast cell lineages. Nature 344, 444-447 (1990).

21. Gutiérrez, L. et al. Gatal regulates dendritic-cell development and survival. Blood 110, 1933-1941 (2007).

22. Sankaran, V. G. et al. Exome sequencing identifies GATA1 mutations resulting in Diamond-Blackfan anemia. J. Clin. Invest. 122, 2439-2443 (2012).

23. Ludwig, L. S. et al. Altered translation of GATA1 in Diamond-Blackfan anemia. Nat. Med. 20, 748-753 (2014).

24. Parrella, S. et al. Loss of GATA-1 full length as a cause of Diamond-Blackfan anemia phenotype. Pediatr. Blood Cancer 61, 1319-1321 (2014). 
25. Mundschau, G. et al. Mutagenesis of GATA1 is an initiating event in Down syndrome leukemogenesis. Blood 101, 4298-4300 (2003).

26. Rainis, L. et al. Mutations in exon 2 of GATA1 are early events in megakaryocytic malignancies associated with trisomy 21. Blood 102, 981-986 (2003).

27. Crispino, J. D. \& Weiss, M. J. Erythro-megakaryocytic transcription factors associated with hereditary anemia. Blood 123, 3080-3088 (2014).

28. Hitzler, J. K., Cheung, J., Li, Y., Scherer, S. W. \& Zipursky, A. GATA1 mutations in transient leukemia and acute megakaryoblastic leukemia of Down syndrome. Blood 101, 4301-4304 (2003).

29. Roberts, I. et al. GATA1-mutant clones are frequent and often unsuspected in babies with Down syndrome: identification of a population at risk of leukemia. Blood 122, 3908-3917 (2013).

30. Gruber, T. A. \& Downing, J. R. The biology of pediatric acute megakaryoblastic leukemia. Blood 126, 943-949 (2015).

31. Gialesaki, S. et al. GATA1s exerts developmental stage-specific effects in human hematopoiesis. Haematologica 103, e336-e340 (2018).

32. Li, Z. et al. Developmental stage-selective effect of somatically mutated leukemogenic transcription factor GATA1. Nat. Genet. 37, 613-619 (2005).

33. Erard, N., Knott, S. R. V. \& Hannon, G. J. A CRISPR resource for individual, combinatorial, or multiplexed gene knockout. Mol. Cell 67, 348-354.e4 (2017).

34. Notta, F. et al. Distinct routes of lineage development reshape the human blood hierarchy across ontogeny. Science 351, aab2116 (2016).

35. Dick, J. E. Stem cell concepts renew cancer research. Blood 112, 4793-4807 (2008).

36. Hu, Y. \& Smyth, G. K. ELDA: extreme limiting dilution analysis for comparing depleted and enriched populations in stem cell and other assays. J. Immunol. Methods 347, 70-78 (2009).

37. Wunderlich, M. et al. Improved multilineage human hematopoietic reconstitution and function in NSGS mice. PLoS ONE 13, e0209034 (2018).

38. Rahmig, S. et al. Improved human erythropoiesis and platelet formation in humanized NSGW41 mice. Stem Cell Rep. 7, 591-601 (2016).

39. Bain, B. J., Clark, D. M. \& Wilkins, B. S. Bone Marrow Pathology (John Wiley \& Sons, 2011).

40. Vyas, P., Ault, K., Jackson, C. W., Orkin, S. H. \& Shivdasani, R. A. Consequences of GATA-1 deficiency in megakaryocytes and platelets. Blood 93, 2867-2875 (1999).

41. Simon, M. C. et al. Rescue of erythroid development in gene targeted GATA1-mouse embryonic stem cells. Nat. Genet. 1, 92-98 (1992).

42. Shimizu, R., Takahashi, S., Ohneda, K., Engel, J. D. \& Yamamoto, M. In vivo requirements for GATA-1 functional domains during primitive and definitive erythropoiesis. EMBO J. 20, 5250-5260 (2001).

43. Halsey, C., Tunstall, O., Gibson, B., Roberts, I. \& Graham, G. Role of GATA$1 \mathrm{~s}$ in early hematopoiesis and differences between alternative splicing in human and murine GATA-1. Blood 115, 3415-3416 (2010).

44. Bhatnagar, N., Nizery, L., Tunstall, O., Vyas, P. \& Roberts, I. Transient abnormal myelopoiesis and AML in Down syndrome: an update. Curr. Hematol. Malig. Rep. 11, 333-341 (2016).

45. Wechsler, J. et al. Acquired mutations in GATA1 in the megakaryoblastic leukemia of Down syndrome. Nat. Genet. 32, 148-152 (2002).

46. Ahmed, M. et al. Natural history of GATA1 mutations in Down syndrome Blood 103, 2480-2489 (2004).

47. Kirsammer, G. et al. Highly penetrant myeloproliferative disease in the Ts65Dn mouse model of Down syndrome. Blood 111, 767-775 (2008)

48. O'Doherty, A. et al. An aneuploid mouse strain carrying human chro7mosome 21 with Down syndrome phenotypes. Science 309, 2033-2037 (2005).

49. Labuhn, M. et al. Mechanisms of progression of myeloid preleukemia to transformed myeloid leukemia in children with Down syndrome. Cancer Cell 36, 123-138.e10 (2019)

50. Draptchinskaia, N. et al. The gene encoding ribosomal protein S19 is mutated in Diamond-Blackfan anaemia. Nat. Genet. 21, 169-175 (1999).

51. Ulirsch, J. C. et al. The genetic landscape of Diamond-Blackfan anemia. Am. J. Hum. Genet. 103, 930-947 (2018).

52. Giri, N., Reed, H. D., Stratton, P., Savage, S. A. \& Alter, B. P. Pregnancy outcomes in mothers of offspring with inherited bone marrow failure syndromes. Pediatr. Blood Cancer 65, e26757 (2018).

53. Ryan, D. E. et al. Improving CRISPR-Cas specificity with chemical modifications in single-guide RNAs. Nucleic Acids Res. 46, 792-803 (2018).

54. Hendel, A. et al. Chemically modified guide RNAs enhance CRISPR-Cas genome editing in human primary cells. Nat. Biotechnol. 33, 985-989 (2015).

55. Mir, A. et al. Heavily and fully modified RNAs guide efficient SpyCas9mediated genome editing. Nat. Commun. 9, 2641 (2018).

56. Gopalappa, R., Suresh, B., Ramakrishna, S. \& Kim, H. H. Paired D10A Cas 9 nickases are sometimes more efficient than individual nucleases for gene disruption. Nucleic Acids Res. 46, e71-e71 (2018).
57. Vakulskas, C. A. et al. A high-fidelity Cas9 mutant delivered as a ribonucleoprotein complex enables efficient gene editing in human hematopoietic stem and progenitor cells. Nat. Med. 24, 1216-1224 (2018).

58. Itoh, K. et al. Reproducible establishment of hemopoietic supportive stromal cell lines from murine bone marrow. Exp. Hematol. 17, 145-153 (1989).

59. Mazurier, F., Doedens, M., Gan, O. I. \& Dick, J. E. Rapid myeloerythroid repopulation after intrafemoral transplantation of NOD-SCID mice reveals a new class of human stem cells. Nat. Med. 9, 959-963 (2003).

60. Brinkman, E. K., Chen, T., Amendola, M. \& van Steensel, B. Easy quantitative assessment of genome editing by sequence trace decomposition. Nucleic Acids Res. 42, e168-e168 (2014)

61. Bae, S., Park, J. \& Kim, J.-S. Cas-OFFinder: a fast and versatile algorithm that searches for potential off-target sites of Cas9 RNA-guided endonucleases. Bioinformatics 30, 1473-1475 (2014).

\section{Acknowledgements}

We thank M. DSouza for support with mouse work; T. Velauthapillai, S. Ng, A. AuYeung, N. Simard and S. Zhao at the SickKids-UHN Flow and Mass Cytometry Facility for assistance with flow cytometry; B. Apresto at the The Centre for Applied Genomics (SickKids) for Sanger sequencing; A. Smith at the Cancer Cytogenetics Laboratory (UHN) for karyotyping; M. Peralta and C. Cimafranca at the Pathology Research Program (UHN) for assistance with histology; M. Bartolini at the Advanced Optical Microscopy Facility (AOMF) for slide scanning; the labs of S. Chan and F. Notta for shared equipment. We thank M. Anders, J. Kennedy and J. Wang for comments on the manuscript. E.W. is supported by a long-term fellowship from the Human Frontier Science Program and a Banting postdoctoral fellowship. L.D.S is supported by National Institute of Health CA034196, AI132963 and DK104218. Work in J.E.D.'s laboratory is supported by grants from the Canadian Institutes for Health Research, Ontario Institute for Cancer Research through funding provided by the Government of Ontario, Terry Fox Foundation Research Institute, Canadian Cancer Society and the University of Toronto's Medicine by Design initiative, which receives funding from the Canada First Research Excellence.

\section{Author contributions}

E.W., J.E.D. and E.L. conceived the project, supervised research, and wrote the paper. E. W., O.I.G. and E.L. analyzed the experiments. E.W., M.A., S.S., L.S. and E.L. performed the in vitro and in vivo experiments. J.L.M. assisted with intrafemoral injections. G.K. assisted with western assay. J.A. assisted with morphology analysis. O.I.G. assisted with single cell in vitro assays and methylcellulose colony formation assays. L.D.S. provided NSGW41 mice.

\section{Competing interests}

The authors declare no competing interests.

\section{Additional information}

Supplementary information is available for this paper at https://doi.org/10.1038/s41467 019-12726-0.

Correspondence and requests for materials should be addressed to J.E.D. or E.R.L.

Peer review information Nature Communications thanks Matthew Porteus and the other, anonymous, reviewer(s) for their contribution to the peer review of this work. Peer reviewer reports are available.

Reprints and permission information is available at http://www.nature.com/reprints

Publisher's note Springer Nature remains neutral with regard to jurisdictional claims in published maps and institutional affiliations.

Open Access This article is licensed under a Creative Commons Attribution 4.0 International License, which permits use, sharing, adaptation, distribution and reproduction in any medium or format, as long as you give appropriate credit to the original author(s) and the source, provide a link to the Creative Commons license, and indicate if changes were made. The images or other third party material in this article are included in the article's Creative Commons license, unless indicated otherwise in a credit line to the material. If material is not included in the article's Creative Commons license and your intended use is not permitted by statutory regulation or exceeds the permitted use, you will need to obtain permission directly from the copyright holder. To view a copy of this license, visit http://creativecommons.org/ licenses/by/4.0/.

(C) The Author(s) 2019 